Article

\title{
On Self-Interference Cancellation and Non-Idealities Suppression in Full-Duplex Radio Transceivers
}

\author{
Areeba Ayesha ${ }^{1}$, MuhibUr Rahman ${ }^{2}\left(\mathbb{D}\right.$, Amir Haider ${ }^{3, *(D)}$ and Shabbir Majeed Chaudhry ${ }^{1, *}$ \\ 1 Department of Electrical Engineering, University of Engineering and Technology, \\ Taxila 47050, Punjab, Pakistan; areebaa80@gmail.com \\ 2 Department of Electrical Engineering, Polytechnique Montreal, Montreal, QC H3T 1J4, Canada; \\ muhibur.rahman@polymtl.ca \\ 3 Department of Intelligent Mechatronics Engineering, Sejong University, Seoul 05006, Korea \\ * Correspondence: amirhaider@sejong.ac.kr (A.H.); shabbir.majeed@uettaxila.edu.pk (S.M.C.)
}

Citation: Ayesha, A.; Rahman, M.; Haider, A.; Majeed Chaudhry, S. On Self-Interference Cancellation and Non-Idealities Suppression in Full-Duplex Radio Transceivers. Mathematics 2021, 9, 1434. https:// doi.org/10.3390/math9121434

Academic Editor:

Alessandro Niccolai

Received: 23 April 2021

Accepted: 15 June 2021

Published: 20 June 2021

Publisher's Note: MDPI stays neutral with regard to jurisdictional claims in published maps and institutional affiliations.

Copyright: (C) 2021 by the authors. Licensee MDPI, Basel, Switzerland. This article is an open access article distributed under the terms and conditions of the Creative Commons Attribution (CC BY) license (https:/ / creativecommons.org/licenses/by/ $4.0 /)$.

\begin{abstract}
One of the major impediments in the design and operation of a full-duplex radio transceiver is the presence of self-interference (SI), that is, the transceiver's transmitted signal, 60-100 dB stronger than the desired signal of interest. To reduce the SI signal below the receiver's sensitivity before coupling it to the receiver, radio frequency (RF)/analog domain cancellation is carried out. Even after SI cancellation to the required level in the analog domain, the residual SI signal still exits and lowers the transceiver's performance. For residual SI cancellation, a digital domain cancellation is carried out. RF impairments are the major obstacle in the residual SI cancellation path in the digital domain. Linearization of RF impairments such as IQ mixer imbalance in the transmitter and receiver chain, non-linear PA with memory, and non-linear LNA are also carried out. Performance evaluation of the proposed techniques is carried out based on SINR, the power of different SI signal components, PSD, output to input relationship, SNR vs. BER, spectrum analyzer, constellation diagram, and link budget analysis. The proposed techniques provide attractive RF/analog SI cancellation of up to 80-90 dB, digital residual SI cancellation of up to 35 to $40 \mathrm{~dB}$, total SI cancellation of up to 110 to $130 \mathrm{~dB}$, and an SINR improvement of up to $50 \mathrm{~dB}$.
\end{abstract}

Keywords: RF/analog cancellation; channel state information at receiver (CSIR); digital SI cancellation; full-duplex (FD); self-interference (SI); residual self-interference; transceiver RF impairments / non-idealities

\section{Introduction}

A full-duplex (FD) communication system represents an attractive substitute to halfduplex (HD) communication systems. FD is a bidirectional communication scheme, which pursues simultaneous uplink and downlink transmission at the same radio frequency band at the same time. In the FD communication system, communication takes place over the same spectral and temporal resources, it has increased spectral efficiency and throughput and shares the single carrier frequency between uplink and downlink. However, simultaneous transmission and reception over the same carrier frequency at the same time results in significant limitations to the FD communication system, i.e., self-interference (SI) signal. The FD communication system has gained many researchers' attention with regard to the next-generation wireless radio communication systems such as 5G. Because of the FD communication system's immense advantages over the HD communication system, researchers are trying to overcome the drawbacks or problems that are causing trouble for successfully implementing the FD communication system's transceivers for communication purposes. In the last few decades, a huge interest from academia has been seen towards FD communication system design, implementation and SI cancellation, i.e., major FD communication system barriers. The FD communication system can mitigate some essential issues such as a hidden terminal problem at the physical layer, persistent issues in the carrier 
sense multiple access control (MAC) networks, and fairness in a network. The FD communication system lowers the transmission delay. It is suitable for implementing feasible cognitive radio to detect and avoid delay, and limit a primary transmission and security of wireless data transfer [1-3]. The FD communication system has some advantages over frequency division duplexing (FDD) and time division duplexing (TDD). These include increased spectral efficiency by almost double, sharing single frequency between uplink and downlink, sensing the network traffic during its transmission, improved throughput, and lowering transmission delay.

Despite these advantages, the practical realization and implementation of FD radio transceivers still have many challenges. Complications arise when co-located transmit and receive antennae (either single antenna or closely spaced two separate antennae) operate on the same carrier frequency at the same time, such as in FD radio transceivers [1,4-10]. A most substantial and prime obstacle to the practical realization and implementation of FD radio transceivers is SI and inter-node interference. SI signal is almost 60-100 dB stronger than the desired received signal. SI signal overshadows the signal being received and highly degrades the receiver sensitivity and link throughput [1]. SI phenomenon is one of the biggest challenges in FD communication systems, stemming from imperfect electromagnetic isolation between transmitter (Tx) and receiver (Rx). Hence, in order for the FD communication system to achieve maximum efficiency and throughput, efficient SI cancellation mechanisms and appropriate antenna isolation is required. These are necessary to reduce the SI signal down to a sufficiently low level, i.e., ideally to the level below the receiver's noise floor and sensitivity level.

FD transceivers capable of overcoming self-interference (SI) have the potential to improve the spectral efficiency, capacity of wireless networks and flexibility of spectrum use. FD can maximize the end-to-end transmission and hence result in extended coverage and enhanced communication system link quality. Regardless of the immense benefits and increased throughput provided by FD technology, a few practical obstacles prevent its widespread deployment, and the industry has shown a very limited and slow adoption, i.e., in terms of commodity in cellular and Wi-Fi devices. An accurate and desired level SI cancellation and suppression of non-idealities of the transceiver's components requires complicated and complex circuity which, in return, impart a huge challenge to fit compactly in small/cellular/smart devices. Currently, FD operation has a major barrier of space constraints and bulky size of circuitry that prevents its usage on end user devices. The FD communication system is extremely energy/power hungry. These are all factors that mean it can consume approximately $40 \%$ additional power in a mobile phone in active RF mode [11]. Moreover, the traffic patterns in higher layers of are highly asymmetric i.e., downlink traffic demand is higher than uplink demand and another major drawback of the FD communication system is that it shows maximum gain and throughput when there is symmetric traffic pattern in higher layers i.e., equal traffic flow in both directions [12]. FD can play a vital role in domain where the traffic is symmetric in nature, and it requires high throughput, as with collaborative sensing for autonomous vehicles.

FD devices has been applied in numerous wireless communication systems such as cognitive radio, cooperative networks, device to device, multi-input multi-output (MIMO) communication and relay networks [13]. As per the report of North Atlantic Treaty Organization's (NATO) Science and Technology Organization, FD technology has the ability to alleviate spectral congestion issues in tactical communications. Similarly, FD technology can be used for the security of civilian wireless communications, i.e., through the use of jamming and receiving wireless signals simultaneously, which can in turn prevent eavesdropping. Moreover, it can also help in the prevention of unauthorized usage of the radio spectrum [14]. The FD communication system can also be used for military and defense purposes such as Radio Detection and Ranging (RADAR) sensing and RF backscatter. 


\subsection{Related Work}

Numerous SI cancellation methods have been proposed to lessen the transceiver's SI effects on the receiver's performance. These SI cancellation methods can be categorized as passive and active cancellation techniques. It has been reported in $[8,15-18]$ that SI signal can be significantly alleviated to the desired level by using the passive SI cancellation technique, also known as antenna cancellation technique. The active SI cancellation technique includes SI cancellation in propagation or analog domain, also known as RF cancellation, as presented in [2,3,19-23], to lessen the SI signal significantly. The residual SI signal cancellation at the receiver chain has been demonstrated in [24,25], as a digital SI cancellation technique. SI, i.e., the transmitter's transmitted signal is at a higher level (dB) than our desired signal of interest. Digital SI cancellation techniques are useless if the receiver gets saturated due to the SI signal. Hence, for SI cancellation to the desired level, passive and analog cancellation techniques are used as pre-cancellation techniques. Effective analog pre-cancellation is an essential prerequisite for the implementation of digital cancellation. However, neither of these techniques alone is enough to mitigate the SI to the desired level fully. Typically, the FD communication system deploys both active and passive cancellation techniques sequentially to significantly reduce the SI signal, i.e., below the receiver's thermal noise floor or sensitivity level.

The implementation of the antenna cancellation technique and analog/RF cancellation does not effectively cancel the SI signal because of imperfect channel estimation between Tx and Rx antennae. Similarly, antenna cancellation and digital cancellation do not effectively mitigate the SI signal because of the limited dynamic range of analog to digital convertor (ADC) in the receiver chain. However, by implementing an antenna cancellation technique combined with noise cancellation and digital interference cancellation, the mitigation of the SI signal up to $60 \mathrm{~dB}$ can be achieved, permitting a node to transmit and receive simultaneously [1]. Upon implementing both active cancellation techniques, i.e., analog/RF cancellation and digital cancellation simultaneously, $60 \mathrm{~dB}$ SI signal mitigation has been stated in [26,27] and 70-73 dB in [6,7]. Antenna separation and both active cancellation techniques (analog and digital) are effective because a reasonable amount of SI signal can be cancelled before it is coupled to the receiver; otherwise, the receiver goes to saturation and this degrades the performance. A maximum of $100 \mathrm{~dB}$ SI cancellation has been reported in the literature up until now, when both passive (antenna) cancellation and active (analog and digital) SI cancellation techniques are used at once [28].

Perfect suppression of SI is impossible in practice; even after applying antenna and analog cancellation techniques, residual SI still exists. The statistical properties of SI are different from those of thermal noise, but they always act in a similar way to noise, and are also treated as noise [29]. Digital cancellation techniques are applied at the transceiver's baseband side and are used to suppress residual SI, i.e., low power multi-path components of SI $[6,7,24,25]$. It is also applied to compensate for non-linear components of the transmitter and receiver front end [30]. Performance of the FD communication system's transceiver is highly degraded due to non-idealities in different transceivers' architecture stages, i.e., Tx and Rx front-end components (low cost, low-quality components) and due to the circuitry used for active and passive cancellation [31,32]. Non-idealities present at the $\mathrm{Tx}$ and $\mathrm{Rx}$ chain include the phase noise of a local oscillator for in-phase and quadrature (IQ) mixer [15,33-37], IQ mismatch (imbalance between gain and phase of I and Q branches of IQ mixer and image components) $[24,38,39]$, receiver noise figure, ADC quantization noise [40] and digital to analog convertor (DAC) and ADC dynamic range [41,42], power amplifier (PA) nonlinearity [3,43,44], and low noise amplifier (LNA) saturation [42,45]. These non-idealities mentioned above are the major and foremost obstacle in perfect SI cancellation, and they cause residual SI. An iterative estimation and cancellation technique for nonlinear in-band full-duplex transceivers with IQ imbalances and amplifier nonlinearities has been presented in [46]. 


\subsection{Contributions}

In order to resolve the problem of SI in the FD communication system, several SIG techniques have been proposed in the past. To reduce the transceiver's SI effects on the receiver's performance, both in the analog and digital domain, i.e., RF/analog and digital SI cancellation, much research have been conducted in the radio layer. Any one of the cancellation techniques alone does not have the capability to provide SI cancellation to the desired level. Moreover, existence of non-idealities in the transceiver's components act as a huge hurdle in mitigation of SI.

In this work, in order to gain high efficiency and SIC to the desired level, different SIC techniques, both in the analog and digital domains, along with linearization techniques of the transceiver's component is presented. This manuscript is focused on the functioning of the FD communication system and mitigation of its impediments at the radio layer. The FD widely linear direct conversion transceiver model is utilized to validate the performance of the proposed methods. The main contribution of this work is setting out the following:

- Three RF/analog SIC techniques, i.e., CSI, GALL filter and Kautz filter for SI cancellation to level below the receiver's thermal noise floor or sensitivity level.

- A digital SIC technique i.e., Kalman filter for residual SI cancellation.

- Linearization techniques for the suppression of the transceiver's component (LNA, PA, IQ mixer) non idealities, i.e.,

$>\quad$ BAFF and BA algorithm for IQ mixer imbalance

$>\quad$ Doherty amplifier technique with feedforward linearization technique and pre-distortion linearization technique for the linearization of PA

$>\quad$ Negative feedback and feedforward linearization technique for LNA.

Metrics used for FD system evaluation are link-budget analysis, power calculation of different SI signal components, and signal to interference plus noise ratio (SINR). Performance analysis of the FD transceiver's transmitter chain components is carried out separately, and results are shown through constellation diagrams and spectrum analyzers. Tx's IQ mixer performance is analyzed through constellation diagrams. PA linearity analysis is performed by examining PA power spectral density (PSD) and its input to output relationship. Unlike the Tx chain, Rx chain performance analysis is carried out by using the metric bit error rate (BER) vs. signal to noise ratio (SNR). LNA linearity analysis is performed separately and examined through PSD and the output vs. input relationship of LNA with and without linearization techniques.

\subsection{Organization of the Paper}

The rest of the paper is organized as follows: the proposed system model and techniques for SIC and the linearization of non-idealities of the transceiver's components are described in Section 2. The simulations, results and discussion for the proposed system and techniques along with comparison of the proposed work with the previous works are described in Section 3. Finally, Section 4 presents the conclusion of our work.

\section{Proposed System Model and Techniques}

The architecture considered for performance analysis and result verification is a wellknown transceiver model, i.e., DC transceiver, and is shown in Figure 1 along with SI cancellation techniques and transceiver component's non-idealities. The RF impairments, which the DC transceiver faces during practical realization and implementation, are IQ imbalance, phase noise, and frequency offset due to local oscillator (LO), non-linear behavior of PA and LNA, and DAC and ADC dynamic range. 


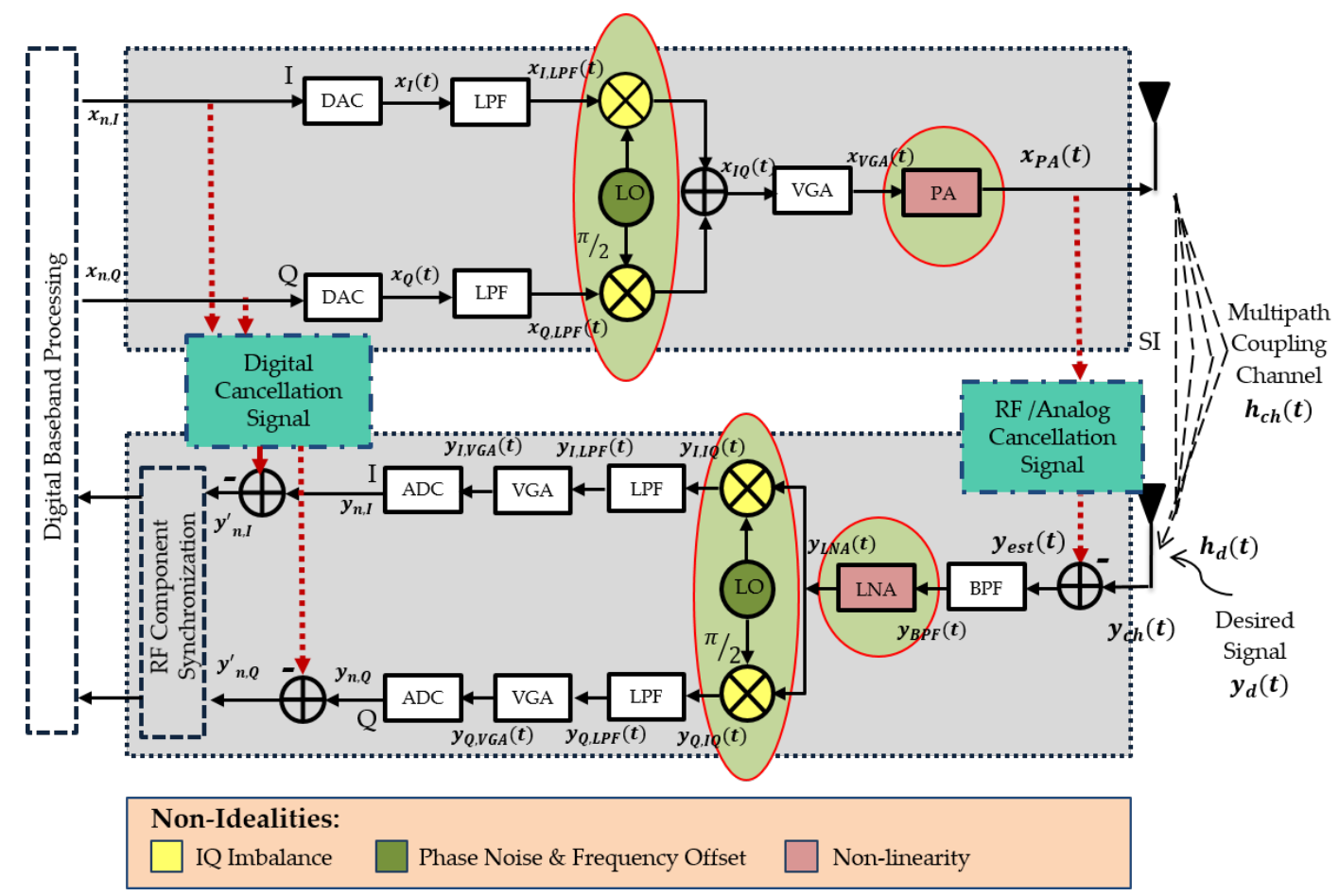

Figure 1. Widely linear direct conversion (DC) full-duplex transceiver.

\subsection{Transceiver's Components Linearization}

The signal that is transmitted after digital to analog conversion (represented by $x_{I}(t)$ and $x_{Q}(t)$ ) and amplification (represented by $\left.x_{P A}(t)\right)$ is the baseband signal $x_{n}$ (represented by its two components $x_{n, I}$ and $x_{n, Q}$ ). DAC and low pass filter (LPF) are considered ideal, and variable gain amplifier (VGA) is omitted from calculations for the sake of simplicity. However, IQ, PA, and LNA nonlinearity is considered. The IQ imbalance problem arises because of a gain and amplitude mismatch between the in-phase and quadrature branches of the IQ mixer. Phase noise and frequency offset also result from IQ mixer's LO IQ mixer mismatch in Tx, and the Rx chain is considered with the help of the image rejection ratio (IRR), as defined by the 3rd generation partnership project (3GPP) long-term evolution-advance (LTE-A) specification, i.e., $25 \mathrm{~dB}$ for both Tx and Rx chain. 3GPP LTE-A system specification limits the minimum image attenuation to $25 \mathrm{~dB}$ or $28 \mathrm{~dB}$ [47]. $25 \mathrm{~dB}$ attenuation is sufficient for the Tx chain.

However, the Rx chain IQ imbalance adds additional image attenuation. IQ imbalance in the Rx chain affects the residual SI signal cancellation. As a widely linear model is used for analysis, so both direct and image components are taken. Image rejection ratio (IRR) determines the quality of mismatch between I and Q branches. PA nonlinearity is modeled as the Hammerstein memory model. Signal after amplification from PA gets coupled to its receiver, and this coupled signal is called an SI signal. The channel between Tx and $\mathrm{Rx}$ is considered a multi-path coupling channel. SI signal is 60-100 dB stronger than our desired signal (the signal which we wish to receive), so it superimposes the desired signal of interest and results in degradation and distortion of the desired signal. SI also causes the saturation of the receiver chain. Hence, it is crucial to mitigate the SI below the sensitivity of the receiver chain before it enters the LNA. Now, what we obtain at the receiver antenna is the signal $y_{c h}(t)$, as shown in Equation (1).

$$
y_{c h}(t)=h_{d}(t) * x_{d}(t)+h_{c h}(t) * x_{P A}(t)+n_{t h}(t),
$$

Here $x_{P A}(t)$ denotes PA output, $x_{d}(t)$ denotes the desired signal, $h_{d}(t)$ denotes desired signal channel, $h_{c h}(t)$ denotes multi-path coupling channel and $n_{t h}(t)$ denotes thermal noise. 


\subsubsection{IQ Mixer Linearization}

A blind adaptive feedforward algorithm (BAFF) [48-50] is implemented for the compensation and estimation of gain and phase mismatch for transmitter chain IQ imbalance. For Rx chain IQ imbalance, a blind adaptive algorithm (BA) [51,52] is implemented. BAFF algorithm is simple and has low complexity. Both BAFF and BA are blind and do not require any pilot symbols or training elements for estimation and compensation. Because of their blind nature, they can be easily incorporated into any transceiver and any communication standard. It does not involve training of the desired signal; instead, only statistical properties are needed. Both schemes do not have any stability or convergence issues. Figure 2 shows the complete block diagram of the IQ mixer with gain and phase mismatch and IQ mixer linearization technique (BAFF and BA algorithm).
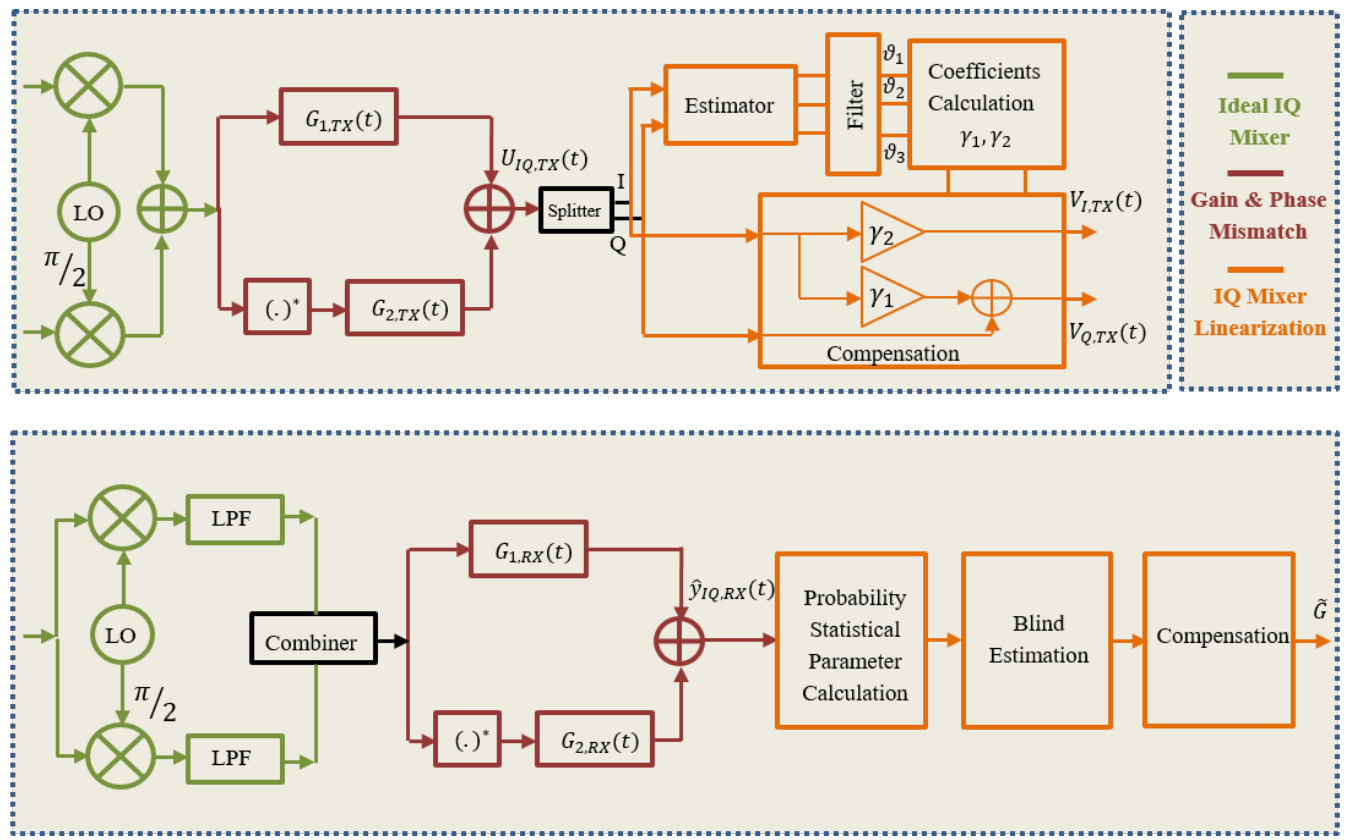

Figure 2. IQ mixer imbalance in transceiver and IQ mixer linearization technique (BAFF and BA algorithm).

\section{Tx: Blind Adaptive Feedforward Algorithm (BAFF)}

For its implementation, three estimators are required to estimate necessary parameters, which involve 1-bit quantization. In the BAFF algorithm, first, we estimate the three parameters $\vartheta_{1}, \vartheta_{2}, \vartheta_{3}$. These three parameters are further used for the calculation of coefficients $\gamma_{1}, \gamma_{2}$ for the compensation of IQ imbalance, and for calculating new gain and phase $\hat{g}$ and $\hat{\varphi}$.

Output after applying BAFF algorithm can be expressed as:

$$
V_{I Q, T X}(t)=\frac{V_{I, T X}(t)+j V_{Q, T X}(t)}{\gamma_{2, T X}}
$$

where,

$$
\begin{gathered}
V_{I, T X}(t)=g_{T X} \gamma_{2, T X} * U_{I, T X}(t) \\
V_{Q, T X}(t)=\gamma_{1, T X} * U_{I, T X}(t)+U_{Q, T X}(t)
\end{gathered}
$$

IQ Mixer coefficients:

$$
\begin{gathered}
\gamma_{1, T X}=\frac{\vartheta_{1, T X}}{\vartheta_{2, T X}}=g_{T X} \sin \left(\varphi_{T X}\right) \\
\gamma_{2, T X}=\sqrt{\frac{\vartheta_{3, T X^{2}-\vartheta_{1, T X}}}{\vartheta_{2, T X}^{2}}}=g_{T X} \cos \left(\varphi_{T X}\right)
\end{gathered}
$$


New estimated gain and phase for Tx chain IQ mixer:

$$
\hat{g}_{T X}=\frac{\vartheta_{3, T X}}{\vartheta_{2, T X}} \text { and } \hat{\varphi}_{T X}=\sin ^{-1}\left(\frac{\vartheta_{1, T X}}{\vartheta_{3, T X}}\right)
$$

For Tx chain IQ mixer:

$$
\begin{gathered}
\vartheta_{1, T X}=-E\left\{\operatorname{sgn}\left(U_{I, T X}(t)\right) U_{Q, T X}(t)\right\} \\
\vartheta_{2, T X}=E\left\{\left|U_{I, T X}(t)\right|\right\} \\
\vartheta_{3, T X}=E\left\{\left|U_{Q, T X}(t)\right|\right\}
\end{gathered}
$$

Rx: Blind Algorithm (BA)

As with the Tx chain IQ mixer, asymmetric IQ imbalance is also modeled in the $\mathrm{Rx}$ chain. The output of Rx IQ mixer after passing through LPF can be expressed as desired/wanted signal and image/unwanted signal, as shown in Equation (3):

$$
\hat{y}_{I Q, R X}(t)=G_{1, R X}(t) * y_{I Q, R X}(t)+G_{2, R X}(t) * y_{I Q, R X} *(t),
$$

where,

$$
\begin{gathered}
G_{1, R X}(t)=\frac{1+g_{R X} e^{-j \varphi_{R X}}}{2} \\
G_{2, R X}(t)=\frac{1-g_{R X} e^{j \varphi_{R X}}}{2}=1-G_{1, R X}{ }^{*}(t)
\end{gathered}
$$

$g_{R X}$ and $\varphi_{R X}$ represents amplitude mismatch and phase mismatch respectively, $y_{I Q, R X}(t)$ and $G_{1, R X}(t)$ are the desired/wanted signal components whereas $y_{I Q, R X}{ }^{*}(t)$ and $G_{2, R X}(t)$ are the image/unwanted signal components.

The above Equation (3) can be expressed in terms of orthogonal frequency division multiplexing (OFDM) symbols as non-negative " $O_{m}(n)$ " and mirror " $O_{-m}(n)$ " OFDM sub-carriers with IQ imbalance after ADC as:

$$
\left[\begin{array}{c}
O_{m}(n) \\
O_{-m}(n)
\end{array}\right]=\left[\begin{array}{cc}
G_{1, R X} & G_{2, R X} \\
G^{*}{ }_{2, R X} & G^{*}{ }_{1, R X}
\end{array}\right]\left[\begin{array}{c}
\hat{y}_{I Q, R X}(n) \\
\hat{y}^{*}-I Q, R X \\
I_{Q}(n)
\end{array}\right]
$$

where, $G=\left[\begin{array}{cc}G_{1, R X} & G_{2, R X} \\ G^{*}{ }_{2, R X} & G^{*}{ }_{1, R X}\end{array}\right]:$ is an interference matrix

" $G$ " can be break into leakage and scaling matrix as:

$$
\hat{G}=\left[\begin{array}{cc}
1 & \frac{G_{2, R X}}{G^{*} 1, R X} \\
\frac{G^{*}}{G_{1, R X}} & 1
\end{array}\right]\left[\begin{array}{cc}
G_{1, R X} & 0 \\
0 & G^{*}{ }_{1, R X}
\end{array}\right]
$$

Here: The first one is the leakage matrix, and the second one is the scaling matrix. The leakage matrix is subjected to IQ mixer imbalance compensation and estimation, whereas the scaling matrix can be cancelled through channel estimation, and $\hat{G}_{q}=\frac{G_{2, R X}}{G^{*}{ }_{1, R X}}$.

OFDM symbols can be reconstructed by using the matrix inversion property, as in realistic case, " $G$ " can never be singular for imbalance OFDM symbols. Here the principal or critical point is gaining knowledge about mixing matrix " $\mathrm{G}$ ".

For the estimation of the " $G$ " matrix and compensation, two probability statistics are used for parameter calculation: (i) cross-correlation b/w OFDM symbols " $E\left\{O_{m}(n) O_{-m}(n)\right\}$ ", and (ii) power of sum of OFDM symbols " $E\left\{\left|O_{m}(n)+O^{*}{ }_{-m}(n)\right|^{2}\right\}$ ". Here an assumption is made.

Assumption:

$E\left\{\hat{y}_{I Q, R X}(n) \hat{y}_{-I Q, R X}(n)\right\}=0$, i.e., uncorrelated OFDM symbols with zero

Further:

$$
G_{1, R X}+G^{*}{ }_{2, R X}=G_{2, R X}+G^{*}{ }_{1, R X}=1
$$


IQ imbalance parameter estimation through BA algorithm can be expressed as:

$$
G_{1, R X} G_{2, R X}=\frac{\sum_{m \epsilon \mathrm{m}} \sum_{n \epsilon \uparrow} E\left\{O_{m}(n) O_{-m}(n)\right\}}{\sum_{m \epsilon \mathrm{m}} \sum_{n \epsilon \mathfrak{\eta}} E\left\{\left|O_{m}(n)+O^{*}{ }_{-m}(n)\right|^{2}\right\}}
$$

And for $\hat{G}_{q}$ calculation:

$$
\widehat{G}_{q}=p_{q}\left(\frac{\sum_{m \epsilon \mathrm{m}} \sum_{n \epsilon \mathfrak{\eta}} E\left\{O_{m}(n) O_{-m}(n)\right\}}{\sum_{m \in \mathrm{m}} \sum_{n \in €} E\left\{\left|O_{m}(n)+O^{*}{ }_{-m}(n)\right|^{2}\right\}}\right)
$$

where,

m: Non-negative Subcarrier indices

n: sample time indices

New estimated gain $\hat{g}_{R X}$ and phase $\hat{\varphi}_{T X}$ can be defined as:

$$
\hat{g}_{R X}=\sqrt{1-4 \operatorname{Re}\left\{G_{1, R X} G_{2, R X}\right\}}
$$

and:

$$
\hat{\varphi}_{T X}=\sin ^{-1}\left\{\frac{-2}{\hat{g}_{R X}} \operatorname{Im}\left\{G_{1, R X} G_{2, R X}\right\}\right\}
$$

Now the compensation matrix can be written as:

$$
\widetilde{G}^{-1}=\frac{1}{\operatorname{Adj} \widetilde{G}}|\widetilde{G}|=\frac{1}{\left|\widetilde{G}_{1, R X}\right|^{2}-\left|\widetilde{G}_{2, R X}\right|^{2}}\left[\begin{array}{cc}
\widetilde{G}^{*}{ }^{1, R X} & -\widetilde{G}_{2, R X} \\
-\widetilde{G}^{*}{ }_{2, R X} & \widetilde{G}_{1, R X}
\end{array}\right]
$$

Compensated OFDM symbols:

$$
\left[\begin{array}{c}
\widetilde{y}_{I Q, R X}(n) \\
\widetilde{y}^{*}-I Q, R X(n)
\end{array}\right]=\widetilde{G}^{-1} \widetilde{G}\left[\begin{array}{c}
\hat{y}_{I Q, R X}(n) \\
\hat{y}^{*}-I Q, R X \\
\end{array}\right],
$$

The imbalance between the I and $Q$ branches of Tx and Rx chain mixer increases with an increase in carrier frequency. A higher constellation is required for achieving high data rates and high operating SNR. To achieve all the above, a high complex analog domain circuit is needed. Hence, to eliminate the IQ imbalance in wireless transceivers with low complexity, digital compensation techniques are developed.

\subsubsection{PA and LNA Linearization}

PAs are the critical component at the transmitter end to manage these entire signals and their specific necessities. PA used must be capable of maintaining high power output, high linearity, and high efficiency, and all of them are inter-related quantities. The primary and foremost requirement at the Tx end is high linearity and efficiency.

Several techniques exist in the literature to satisfy the efficiency and linearity tradeoff. Most of these techniques are characterized based on action to reduce or avoid distortion in a non-linear PA. In this research study, the Doherty technique [53-55] combined with one of the linearization techniques is used for efficiency and linearity improvement. The Doherty technique is considered a mature technique and is used for efficiency enhancement.

Doherty technique operation depends on two different transfer function regimes containing two parallel PA, i.e., carrier PA (CPA) and peaking PA (PPA). One is called a low-power and the other as a high-power regime. Low-power is also defined as a linear regime, and high power is a non-linear regime. By combining the two PAs, the linear range increases where the Doherty amplifier operates. Doherty amplifier has a highly complex structure in practice, but it can be endured over its flexible architecture and highefficiency performance, and this amplifier's topology is the fundamental reason behind 
high efficiency. CPA and PPA are ideal current generators with zero reactive freeloading. Load impedance of CCA is increased while PPA load impedance is decreased as compared to CCA. No extra or additional circuit is required for any kind of controlling operation. Sufficient bandwidth (BW) is provided, i.e., up to $20 \mathrm{MHz}$, but is limited to only a single frequency or single band of operation.

For linearity improvement, two different linearization techniques are used combined with the Doherty technique, i.e., the pre-distortion technique [56,57] that avoids distortion and the feedforward technique that reduces the distortion being produced by non-linear PA.

Similarly, for the linearization of LNA, two techniques are implemented, i.e., negative feedback and feedforward [58]. In both LNA and PA, the feedforward linearization technique first compares the attenuated amplifier output with amplifier input, and generates the distortion signal. The distorted signal is then combined destructively at the amplifier output with the help of an auxiliary amplifier, and the resultant signal is a distortion-free signal. However, it can remove or reduce one type of harmonics at a time, i.e., to remove second and third order harmonics. The feedforward technique required an additional degree of freedom, and hence required an additional auxiliary amplifier path. The auxiliary amplifier path power efficiency is decreased, but still provides a reasonable amount of linearization at the $\mathrm{BW}$ range of 3-50 MHz. The negative feedback linearization technique improves $A_{I I P 2}$ and $A_{I I P 3}$ (second and third intercept points) and hence increases the linear operation range of the amplifier. Figure 3 shows the complete block diagram of PA linearization with the efficiency improvement technique. The LNA with negative feedback and feedforward linearization techniques is depicted as well.
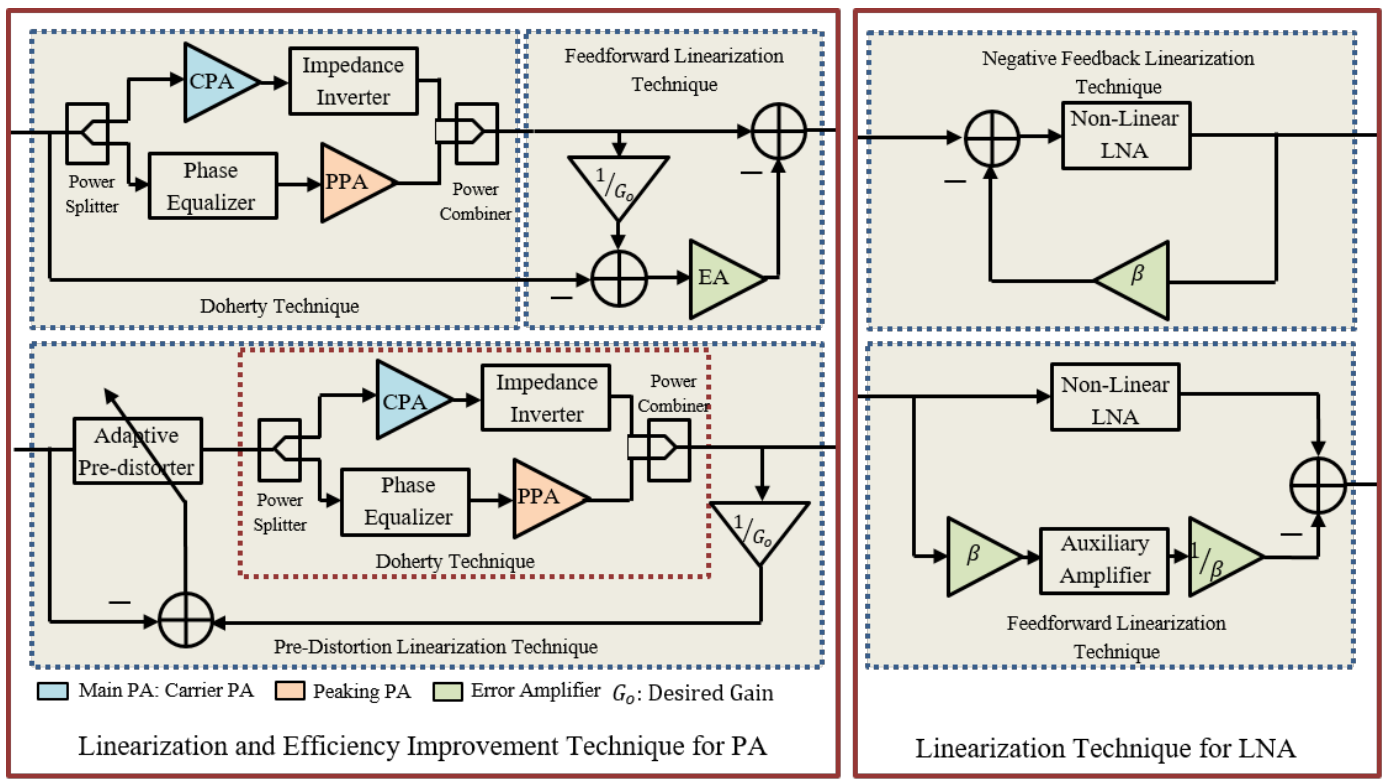

Figure 3. Linearization and efficiency improvement techniques for PA in transmitter RF front end, and linearization techniques for LNA in receiver RF front end.

\subsection{SI Cancellation Techniques}

\subsubsection{Analog/RF SI Cancellation Techniques}

Several analog/RF cancellation techniques exist in the literature, as described in the previous section, to suppress the SI. After applying RF/analog cancellation techniques, the signal at the input of LNA can be described as:

$$
y_{R F}(t)=y_{c h}(t)-y_{e s t}(t)
$$


where " $y_{\text {est }}(t)$ " is the estimated signal attained from RF/analog cancellation techniques and can be expressed as:

$$
y_{\text {est }}(t)=h_{\text {est }}(t) * x_{P A}(t)
$$

As stated above, the significant bottleneck in the efficiency and performance of FD transceivers is the self-interfering signal, i.e., coupling of transmit signal to its Rx. The critical challenge is to cancel the SI signal with one part per billion accuracies, and to cancel the effect of the SI signal over the desired signal up to $100 \%$ approximately. To achieve this accuracy to be the replica of the transmitted signal, SI signal is needed. It is difficult to replicate the SI signal because of multi-path channel effects or the signal from the nearby environment. Two automated operations are required to eliminate the SI in the analog domain efficiently: (1) sensing the self-interfering signal at the Rx, and (2) using adaptive integrated procedure or filter to cancel SI signal to a higher degree of accuracy.

Reliable FD communication systems need to develop an algorithm that can control SI, which is altered by the environment, i.e., reflections from nearby buildings and multi-path channel effects.

\section{Channel State Information (CSI)}

Tx does not have any knowledge about the signal being transmitted over the channel. The signal that a Tx knows is a baseband signal (the signal that is sent after digital to analog conversion and amplification) and the signal present at the output of the PA (the signal before transmission over the channel). Due to the multi-path coupling channel between $T x$ and $R x$, the $R x$ receives multiple copies of the transmitted signal, i.e., delayed, attenuated, and phase-shifted copies of the transmitted signal. Here, multi-path coupling channel characteristic and statistical information is unknown to the Rx [59]. This statistical information of the multi-path coupling channel is crucial for the $\mathrm{Rx}$ to boost the received signal or to remove the SI signal. The knowledge about channel statistical information, i.e., fading distribution type, average channel gain, line of sight (LOS) component, spatial correlation, and impulse response, is termed as channel state information (CSI) [60]. CSI depicts the flow of the signal and how it propagates from the Tx end to the Rx end.

CSI illustrates the properties of the communication link between Tx and Rx. CSI also includes the information about the statistics of received interferences and thermal noise. Using CSI in wireless communication systems aims to improve the channel's capacity, maximize the SNR, and minimize the BER at the detection stage. Primarily, CSI is categorized as: (1) CSI is known to both Tx and Rx (i.e., channel state information at the Tx (CSIT) and channel state information at Rx (CSIR)); (2) CSI is known to Rx only and Tx is unaware of it (i.e., (CSIR)); and (3) both Tx and Rx are unaware of CSI. CSIR is obtained on the Rx side and is used at the Tx side through either the reciprocity principle or feedback method. CSI helps the Tx or Rx adapt to a current channel state for reliable communication with a high data rate. As the channel is primarily a time-varying entity, its statistical information varies with time, so CSI must be predicted on a short-term basis, i.e., must be predicted after a regular interval or period. Usually, a training-based approach is used for estimating the current state of the channel. This approach is considered a popular approach for channel estimation, but the significant overhead of this approach is reduced transmission efficiency [61]. In this approach, CSI is performed using the training period in which a known training sequence or pilot is transmitted over the channel. The required channel matrix is estimated by knowing the transmitted signal and the received antenna's signal. Tx does not directly access CSI; hence, mainly, CSI is utilized at the Rx side, quantized, and fed back to the Tx. Perfect CSI holds when the channel remains static, or the variations are minimal compared to the transmission rate.

Separate transmit and receive antennae are used, and the desired signal of infinitesimal strength compared to self-interfering signal is considered for analysis, as shown in Figure 4. The signal at the Rx antenna " $Y$ " can be expressed as: 


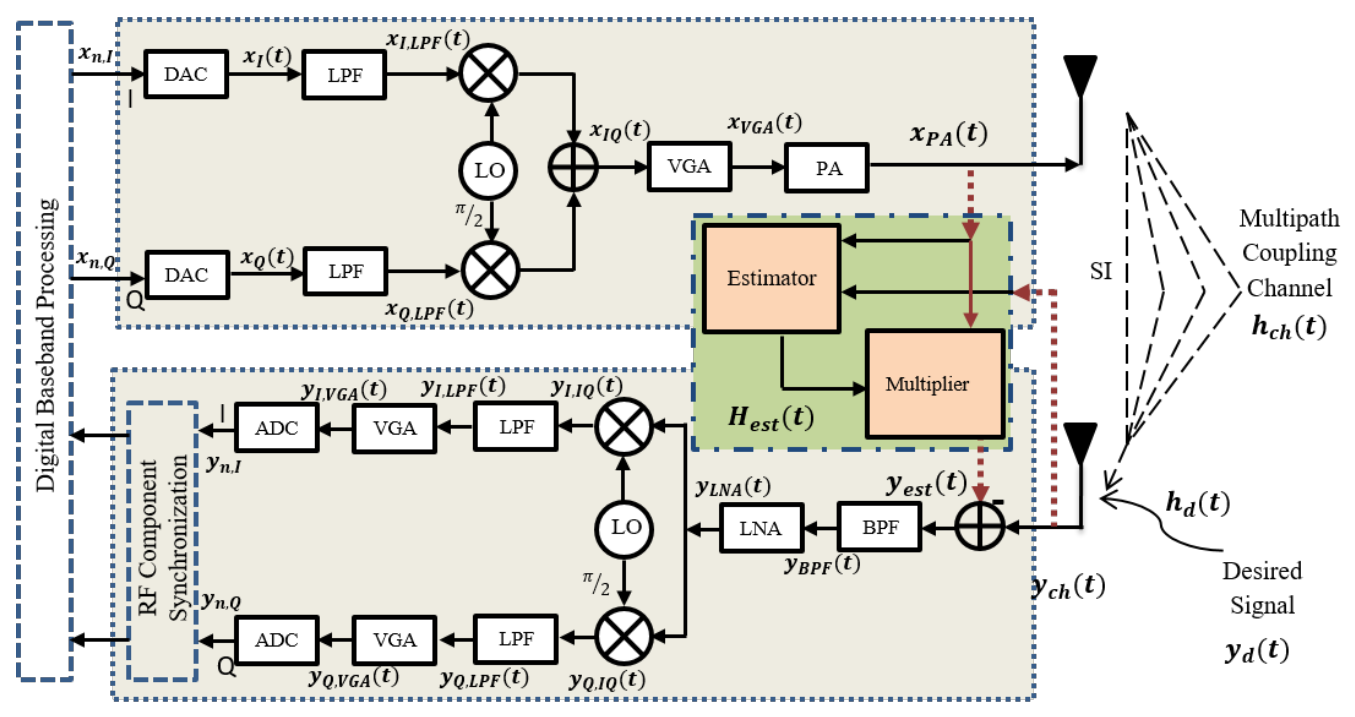

Figure 4. Analog/RF SI cancellation using CSI technique.

$$
\begin{gathered}
Y=Y_{S I}+Y_{D}+W \\
Y=H_{S I} X_{P A}+H_{D} X_{D}+W
\end{gathered}
$$

Her,e $Y_{S I}$ is the SI signal, $Y_{D}$ is the desired signal, and $W$ is the AWGN noise with mean zero and variance $\sigma_{W}^{2}$.

Channel State Information at Rx (CSIR)

During the phase of obtaining CSI at the Rx side, the training sequence is transmitted over the channel and channel $H_{S I}$ is estimated from the knowledge of the received signal " $Y$ " and the training sequence " $P$ ". Let the training sequence be $p_{1}, p_{2}, \ldots \ldots \ldots, p_{T}$, where $P$ is transmitted over the channel. The statistics of the received signal can be described as:

$$
Y=H_{S I} P+W
$$

Here, $Y=\left[y_{1}, y_{2}, \ldots \ldots ., y_{T}\right], P=\left[p_{1}, p_{2}, \ldots \ldots ., p_{T}\right]$ and $W=\left[w_{1}, w_{2}\right.$, $\left.\ldots \ldots \ldots, w_{T}\right]$, " $\mathrm{T}$ " is the length of the training sequence, and " $\mathrm{D}$ " is the length of the data sequence. The total training interval is defined as $L=T+D$ and total time spent during training is defined as duty cycle factor $=\eta=1-\frac{T}{L}$.

Now, if each sub-carrier is orthogonal to the other, then the training symbols for $\mathrm{N}$ sub-carriers can be represented in matrix form as:

$$
P=\left[\begin{array}{ccccc}
p[0] & 0 & & \ldots & 0 \\
0 & p[1] & 0 & \ldots & \vdots \\
\vdots & 0 & \ddots & \ddots & \vdots \\
\vdots & \vdots & & & \vdots \\
0 & \ldots & \ldots & p[N-1]
\end{array}\right] \text {, }
$$

Here, $E\{p[i]\}=0$ is the mean of $i$ th sub-carrier and $\operatorname{Var}\{p[i]\}=\sigma_{i}^{2}$ is the variance of $i$ th sub-carrier, where $i=0,1, \ldots \ldots \ldots, N-1$.

The estimators used for channel estimation to obtain CSI at the Rx side are least square (LS) and minimum mean square error (MMSE) channel estimators. Estimated mean square error (MSE) is proportional to the trace of $P^{H} P$ and the MSE is minimized when $P^{H} P=T I$, i.e., $\operatorname{tr}\left(P^{H} P\right)$ is scaled identity matrix and MSE can also be described as $M S E=\frac{\sigma_{W}^{2}}{\sigma_{i}^{2}}$. Now, the CSI obtained from the training phase is further utilized for the cancellation of SI. As 
the channel is assumed to be static, it remains constant for transmission duration. Here in our transceiver model, CSI gained at the receiver side can be used on the Tx side without any overhead of extra circuitry. Once CSI is obtained, SI can be cancelled by a satisfactory amount given by equations below:

$$
\begin{gathered}
Y=Y_{S I}+Y_{D}+W-Y_{e s t}, \\
Y=\left(H_{S I}-H_{e s t}\right) X_{P A}+H_{D} X_{D}+W,
\end{gathered}
$$

where $Y_{\text {est }}=H_{\text {est }} X_{P A}$.

Hence after estimation of SI signal using CSI and cancellation of estimated SI signal from the total received signal, the signal left at Rx side can be expressed as:

$$
y_{R F .}(t)=h_{d}(t) * x_{d}(t)+\left(h_{c h}(t)-h_{\text {est }}(t)\right) * x_{P A .}(t)+n_{t h}(t),
$$

Blind Estimation

Blind estimation deals with the issues of estimating an unknown signal or input at the receiver side. Input that suffered from phase and gained attenuation due to multi-path fading channel. For the proper equalization and estimation of the channel, the receiver does not require any training sequences from the transmitter. Blind estimation primarily relies on higher-order statistics (HOS) and temporal or spatial oversampling. Many HOS-based blind approaches for blind estimation exist, i.e., Laguerre filter, Kautz filter, and gradient adaptive Laguerre lattice (GALL) filter, etc. [62].

As stated above, the Rx has no prior knowledge about the signal being transmitted over the channel. Due to the multi-path channel and reflection from the nearby environment, the Rx receives multiple copies of the transmitted signal, i.e., delayed, attenuated, and phase-shifted copies of the transmitted signal. To estimate and segregate the transmitted signal from the Rx side, two filters are used for blind estimation: (i) Kautz filter and (ii) GALL filter. After the estimation, segregation, and recovering of the SI signal at the Rx side, it is subtracted from the total received signal. This can be expressed as

$$
\begin{aligned}
& Y=\left(Y_{S I}+Y_{D}+W\right)-Y_{\text {Kautz }}, \\
& Y=\left(Y_{S I}+Y_{D}+W\right)-Y_{G A L L},
\end{aligned}
$$

Here, $Y_{S I}$ denotes SI signal, $Y_{D}$ denotes the desired signal, $W$ indicates additive white Gaussian noise (AWGN) with mean zero and variance $\sigma_{W}^{2}$ and $Y_{G A L L}$, and $Y_{\text {Kautz }}$ denotes the estimated, segregated, and recovered SI signal.

\section{GALL Filter}

A GALL filter is a filter in which a gradient-based approach is used for pole adaption [63]. Pole adaption using a gradient-based method is a straightforward and less complicated technique. Hence, this makes GALL filter implementation easy and straightforward. A GALL filter is used because it wraps time in filter coefficients, i.e., requires less time to calculate and obtain filter coefficients. It also has a lower delay, reduced-order modeling, and requires a smaller number of coefficients and fewer computational operations [64]. It has a fast convergence response and can attain stability in less time. The GALL filter contains an additional free parameter, i.e., a free variable for the independence of memory and the number of adaptive coefficients. Thus, the GALL filter can easily model an extended response filter by using relatively few parameters. Both perform well in steady-state and under tracking conditions [65]. Here, in this paper, for pole adaption, a sign-based gradient algorithm is used.

The transfer function of $n$th order Laguerre filter:

$$
L F_{n}(z, u)=L(z, u) \sum_{m=0}^{n} w_{n, m} A^{m}(z, u)
$$


Laguerre filters have an all-pass filter $A^{m}(z, u)$ with a low pass pre-processing filter stage $L(z, u) . w_{n, i}$ shows adaptive coefficients of the filter. The Laguerre filter's correlation matrix can be expressed as:

$$
x_{m}(t, u)=L(q, u) A^{m}(q, u) x(t)
$$

Here, $x_{m}(t, u)$ represents a Toeplitz matrix. For a lattice description of the Laguerre filter, consider a lattice predictor that transforms the filtered reference signal " $v(t, u)$ " into orthogonal filter backward prediction errors " $\hat{b}_{m}(t, u)$ ", $0<m<M-1$, where $\mathrm{M}$ is the order of lattice predictor and $\hat{h}(t, u)$ will be the estimated secondary path. The filtered reference signal " $v(t, u)$ " can be expressed as:

$$
v(t, u)=x(t, u) * \hat{h}(t, u)
$$

Lattice backward and forward prediction error orthogonalization can be obtained as per Figure 5 and can be defined as:

$$
\begin{gathered}
\hat{f}_{m+1}(t, u)=\hat{f}_{m}(t-1, u)-\kappa_{m}(u-1) \hat{b}_{m}(t-1, u) \\
\hat{b}_{m+1}(t, u)=\hat{b}_{m}(t-1, u)-\kappa_{m}(u-1) \hat{f}_{m}(t, u)
\end{gathered}
$$

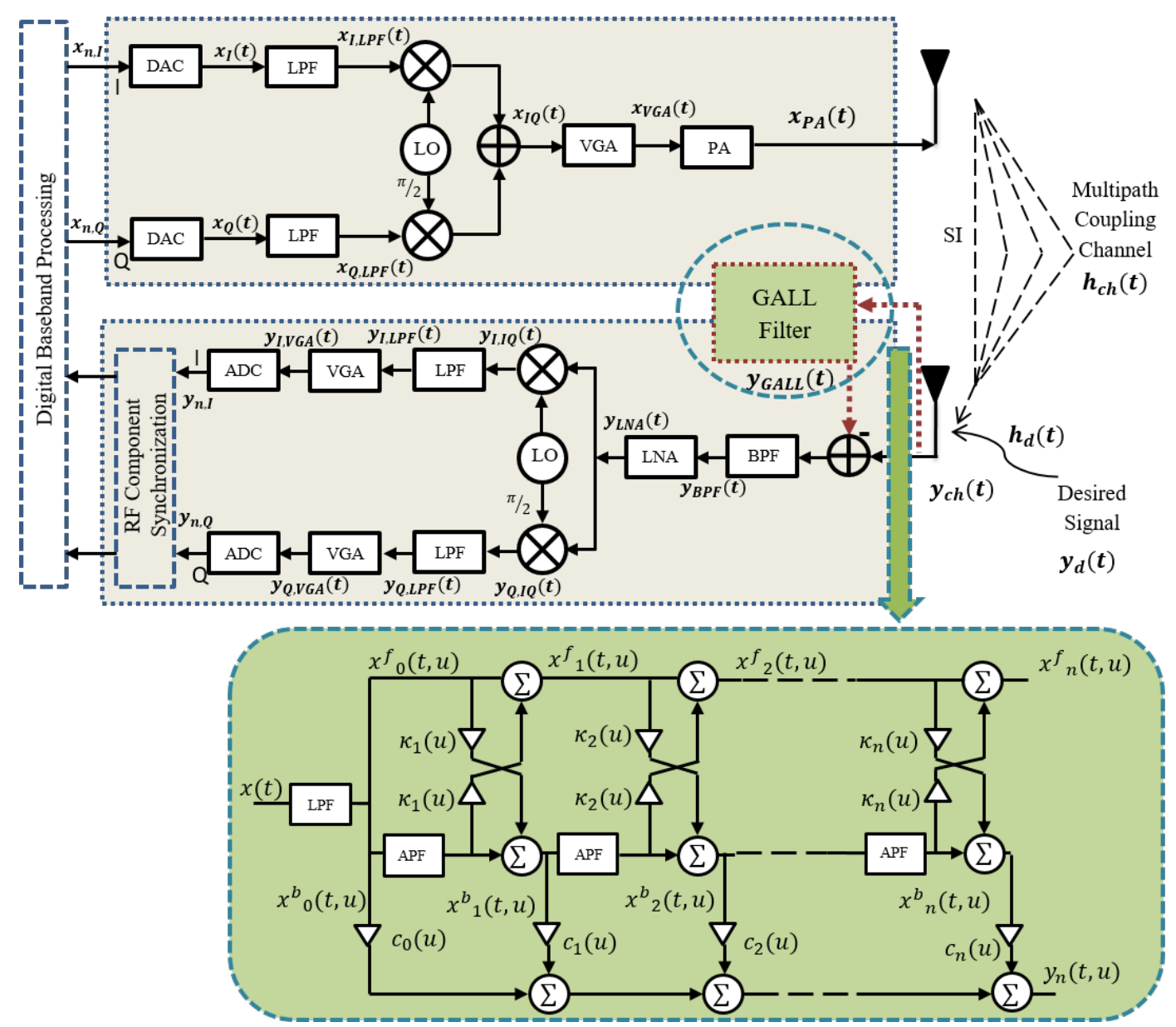

Figure 5. Analog/RF SI cancellation using GALL filter.

The assumption for orthogonality:

$$
E\left\{\hat{b}_{i}(t, u) \hat{b}_{j}(t, u)\right\}=0 \forall i \neq j
$$


The adjustment of $\kappa_{m}(u)$ is carried out as follows by the recursive formula:

$$
\kappa_{m}(u)=\kappa_{m}(u-1)+\frac{\bar{\mu}}{\epsilon_{m}(t, u)+\delta}\left[\hat{f}_{m+1}(t, u) \hat{b}_{m}(t-1, u)+\hat{f}_{m}(t, u) \hat{b}_{m+1}(t-1, u)\right]
$$

where $\bar{\mu}$ is a step size parameter and $\delta$ is a regularization constant, and the power of both forward and backward prediction error of the $m$ th stage can be defined as:

$$
\epsilon_{m}(t, u)=\beta \epsilon_{m}(t-1, u)+(1-\beta)\left[\hat{b}_{m}^{2}(t, u)+\hat{f}_{m}^{2}(t, u)\right]
$$

where $\beta$ is the smoothing parameter.

In the form of correlation matrix lattice, backward and forward prediction error can be expressed as:

$$
\begin{aligned}
& x_{m+1}^{f}(t, u)=x_{m}{ }^{f}(t, u)+\kappa_{m+1}(u) A(q, u) x_{m}{ }^{b}(t, u) \\
& x_{m+1}^{b}(t, u)=A(q, u) x_{m}^{b}(t, u)+\kappa_{m+1}(u) x_{m}{ }^{f}(t, u)
\end{aligned}
$$

where,

$$
\kappa_{m+1}(u)=-\frac{E\left[A(q, u) x_{m}^{b}(t, u) x_{m}^{f}(t, u)\right]}{\sigma_{m}{ }^{2}(u)}
$$

With filtered prediction error:

$$
\begin{gathered}
{\sigma_{m}}^{2}(u)=E\left[x_{m}^{b}(t, u) x_{m}^{b}(t, u)\right]=E\left[x_{m}^{f}(t, u) x_{m}^{f}(t, u)\right] \\
\sigma_{m+1}{ }^{2}(u)=\left[1-\kappa_{m+1}{ }^{2}(u)\right]{\sigma_{m}}^{2}(u)
\end{gathered}
$$

and:

$$
c_{m}(u)=\frac{E\left[y(t) x_{m}^{b}(t, u)\right]}{\sigma_{m}^{2}(u)}
$$

$y(t)$ is the desired output, and $x_{m}(t, u)$ signal is considered linearly independent. Now the prediction error can be defined as:

$$
\epsilon_{m}(t, u)=E\left[y^{2}(t)\right]-\sum_{m=0}^{n} \sigma_{m}^{2}(u) c_{m}^{2}(u)
$$

and in the normalized form:

$$
J_{m}(t, u)=\frac{\epsilon_{m}(t, u)}{E\left[y^{2}(t)\right]}
$$

In the GALL filter, the expectation operator is defined with the help of exponential forgetting window $0<\gamma \leq 1$, as:

$$
E_{\gamma}(x(t))=\left\{\begin{array}{c}
0 t<0 \\
\gamma E_{\gamma}[x(t-1)]+x(t) t>0
\end{array}\right.
$$

Here, all the terms involved in expectation are dependent upon time and result in a time-variant system. In the GALL filter, correct pole adaption " $u$," i.e., change in pole direction, the time-variant approach is used in which all the parameters are estimated on a sample-by-sample basis, and for the sign verification of $c_{m}(u)$ and $c_{m+1}(u)$, the adaption rule is:

$$
u(t+1)=u(t)+\alpha(t) \operatorname{sign}\left[c_{m}(t, u) c_{m+1}(t, u)\right]
$$

Additionally, $\alpha(t)$ should be very small, i.e., order of $10^{-6}$. Any change in " $u$ " will result in a transient state in the GALL filter algorithm, increasing error. GALL filter operates only in the stationary case, so rapid adaption of poles is also worthless, and secondly as 
$|u| \rightarrow 0$, the value of $\alpha(t)$ must decrease. Coefficients of the filter are adapted to diminish the power of the estimated error.

$$
\epsilon_{m}(t, u)=\epsilon_{m-1}(t, u)+h(t, u) *\left[w_{m}(t, u) b_{m}(t, u)\right]
$$

and:

$$
w_{m}(t, u)=w_{m-1}(t, u)-\frac{\mu}{\sigma_{b_{m}}{ }^{2}(t, u)+\delta} \hat{b}_{m}(t, u) \epsilon_{m}(t, u)
$$

Now the updated coefficient equation is defined as:

$$
w(t, u)=w(t-1, u)-\mu(t, u) \sum^{-1}(t, u) \hat{b}(t, u) \epsilon_{m-1}(t, u)
$$

and hence the residual error can be calculated as follows:

$$
\epsilon_{m-1}(t, u)=d(t, u) *\left[w^{T}(t-1, u) b(t, u)\right]
$$

In the next stage, the estimated transmitted signal is used to cancel SI from the received signal to obtain the desired one. Let the estimated SI signal from the GALL filter be $y_{G A L L}(t)$ and error be $\varepsilon_{G A L L}(t)$ now the signal at receiver after analog/RF cancellation by GALL filter can be expressed as:

$$
y_{R F}(t)=\left(y_{c h}(t)-y_{G A L L}(t)\right)+\varepsilon_{G A L L}(t)
$$

As can be seen from Figure 6, GALL filter error " $\varepsilon_{G A L L}(t)$ " decays at a very rapid pace and approaches to zero within a time of about $50 \mu \mathrm{s}$.

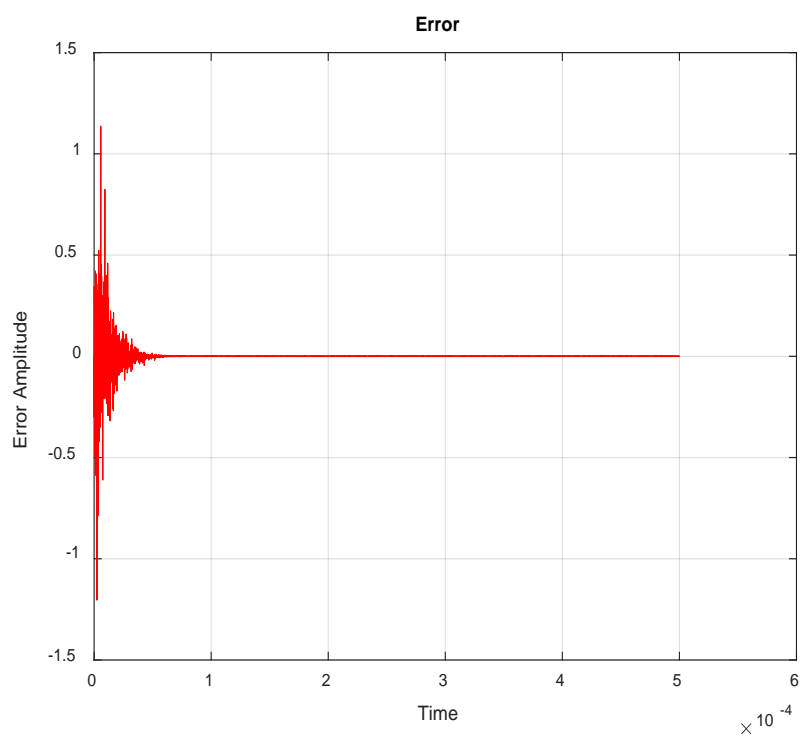

Figure 6. Error analysis of GALL filter.

\section{Kautz Filter}

The Kautz filter is an infinite impulse response (IIR) based tapped delay line transversal filter. It can be well-defined by a set of stable poles and a corresponding set of tapoutput weights. It offers valuable means for optimization and control. It provides linear in-parameter models and adaptive filtering for both non-fixed and fixed structures and various systems identification and approximation schemes [66]. Kautz filter designing can be seen as two different aspects of the optimizing system model and its equalization: (i) choosing an optimal set of poles of Kautz filter $\left\{z_{j}\right\}$, and (ii) evaluating a corresponding optimal set of Kautz filter tap coefficients or weights $\left\{w_{i}\right\}$. These are the two parameters (poles 
and tap coefficients or weights) that determine the filter structures. In signal processing terms, a generic form of the Kautz filter function as per Figure 7 can be described by

$$
H(z)=\sum_{i=0}^{N} w_{i} G_{i}(z)
$$

where,

$$
G_{i}(z)=\frac{\sqrt{1-z_{i} z_{i}^{*}}}{z^{-1}-z_{i}^{*}} \prod_{j=0}^{i} \frac{z^{-1}-z_{j}^{*}}{1-z_{j} z^{-1}}
$$

Here, $i=\{0,1, \ldots, N\}$ and $G_{i}(z)$ is determined by the chosen optimal set of poles $\left\{z_{j}\right\}_{j}^{N}=0$ such that $\left|z_{j}\right|<1$. The following assumptions are made for modeling a real response: (i) poles should be real or (ii) poles occur in complex conjugate pairs.

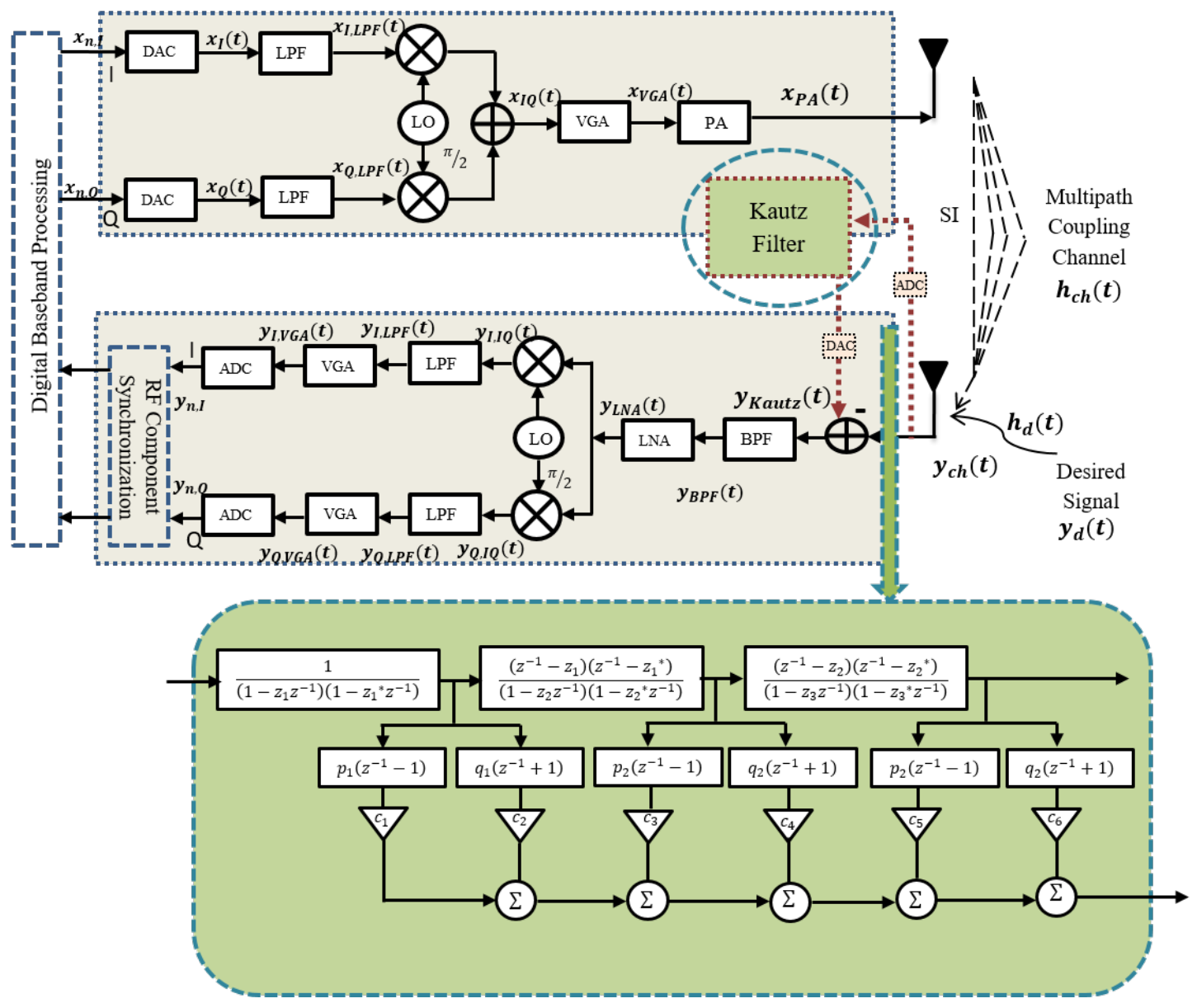

Figure 7. Analog/RF SI cancellation using the Kautz filter.

Normalization terms of the Kautz filter are determined by the corresponding optimal pole pair $\left\{z_{j}, z_{j}^{*}\right\}$ and are described as:

$$
\begin{aligned}
& p_{i}=\sqrt{\frac{\left(1-p_{i}\right)\left(1+p_{i}-r_{i}\right)}{2}}, \\
& q_{i}=\sqrt{\frac{\left(1-p_{i}\right)\left(1+p_{i}+r_{i}\right)}{2}},
\end{aligned}
$$

where $r_{i}=-2 \operatorname{Re}\left\{z_{i}\right\}$ and $p_{i}=\left|z_{i}\right|^{2}$ 
Here, pole adaption is carried out using the BU method. This method is used because of its capability of producing stable and accurate poles, and this method operates in the time domain for signal processing.

\subsubsection{Digital SI Cancellation Techniques}

In a wireless communication system, accurate estimation of desired signal strength is critical [67]. Estimating the desired signal is defined as the process of characterizing the effects of the physical channel, i.e., the transceiver's components and the wireless channel between Tx and Rx on the desired input sequence [68]. Perfect suppression of SI is impossible in practice; even after applying antenna and analog cancellation techniques, residual SI still exists. The statistical properties of SI are different from those of thermal noise, but it still acts in a similar way to noise and is also treated as noise. Digital cancellation techniques are applied at the baseband side of the transceiver and are used to suppress residual SI, i.e., low power multi-path components of SI $[6,7,24,25]$. They are also applied to compensate for non-linear components of the Tx and Rx front end [30]. The performance of the FD transceiver is highly degraded due to non-idealities in different stages of transceiver's architecture, i.e., Tx and Rx front-end components (low cost, low-quality components) and due to the circuitry used for active and passive cancellation.

In this research study, after applying linearization techniques, digital SI cancellation is performed, to mitigate residual SI. Linearization techniques are used to strengthen the residual SI cancellation. Non-linear transmitter and receiver chain components degrade the transceiver's performance and affect the cancellation of residual SI or make residual SI mitigation difficult. In this section, the technique for residual SI cancellation in the digital domain will be addressed. The method used for residual SI cancellation in the digital domain is the Kalman filter. In our previous work [69], we investigated the performance and digital SI cancellation using the extended Kalman filter (EKF) and achieved SINR improvement of about $90 \mathrm{dBm}$.

\section{Kalman Filter}

The Kalman filter is one of the most significant discoveries of the 20th century. One of its most promising and great immediate applications is controlling the complex dynamic systems. It propagates the current state of a dynamic system that is extremely important for the statistical analysis of a system. The propagated information includes the statistical properties of random dynamic perturbations and the influence of all the past measurements [70]. The Kalman filter utilizes the recursive structure, and hence helps in real-time execution. It is a statistical technique. It effectively describes the random forms of experimental measurements. The Kalman filter has relatively low complexity and is a discrete-time linear, finite dimension system [71]. The Kalman filter is usually applied to non-linear systems, and it does not require that the system, i.e., dynamic or random systems under analysis, to have static properties. It involves estimating the system's initial state, and the initial state is obtained by detecting it. The Kalman filter is used most solely for two purposes: (i) estimation of desired signal and (ii) estimator's performance analysis [70]. The Kalman filter is habitually applied to real-time applications because of its robustness and ability to deal with unknown signals. In the Kalman filter, mostly dynamic disturbances are modeled as additional unknown parameters or signals [72]. The Kalman filter has fewer computational requirements, it smoothes the noisy data, and estimates the parameters of interest [73].

Our purpose for applying the Kalman filter as a digital cancellation technique at the digital side of the transceiver is to construct an estimator that can accurately estimate the SI signal. This mitigates the residual SI signal before the digital signal processing of the desired signal, as shown in Figure 1. The Kalman filter provides the best estimate of $x_{n, I}(n)$ and $x_{n, Q}(n)$ at the Rx side through estimator of the form:

$$
\hat{y}_{n, I}^{\prime}(n)=c_{k, I}(n) x_{n, I}(n)+c_{k, I}(n-1) x_{n, I}(n-1)+\ldots+c_{k, I}(1) x_{n, I}(1),
$$




$$
\hat{y}_{n, Q}^{\prime}(n)=c_{k, Q}(n) x_{n, Q}(n)+c_{k, Q}(n-1) x_{n, Q}(n-1)+\ldots+c_{k, Q}(1) x_{n, Q}(1),
$$

where $\hat{y}_{n, I}^{\prime}(n)$ and $\hat{y}_{n, Q}^{\prime}(n)$ are the estimated symbols and $\left\{c_{k, I}(l)\right\}$ and $\left\{c_{k, Q}(l)\right\}, l=$ $n, n-1, \ldots, 1$ are the coefficients to be adapted to the knowledge of the model, " $n$ " represents the total number of symbols.

The Kalman filter involves two successive stages:

(i) Prediction stage:

$$
\begin{gathered}
\hat{y}_{n, I}^{\prime}(n \mid n-1)=\varnothing_{I}(n-1) \hat{y}_{n, I}^{\prime}(n-1 \mid n-1)+\Psi_{I}(n-1) U_{I}(n-1), \\
\hat{y}_{n, Q}^{\prime}(n \mid n-1)=\varnothing_{Q}(n-1) \hat{y}_{n, Q}^{\prime}(n-1 \mid n-1)+\Psi_{Q}(n-1) U_{Q}(n-1),
\end{gathered}
$$

(ii) Updating stage:

$$
\begin{gathered}
\hat{y}_{n, I}^{\prime}(n \mid n)=\hat{y}_{n, I}^{\prime}(n \mid n-1)+k_{I}(n)\left(x_{n, I}(n)-H_{I}(n) \hat{y}_{n, I}^{\prime}(n \mid n-1)\right), \\
\hat{y}_{n, Q}^{\prime}(n \mid n)=\hat{y}_{n, Q}^{\prime}(n \mid n-1)+k_{Q}(n)\left(x_{n, Q}(n)-H_{Q}(n) \hat{y}_{n, Q}^{\prime}(n \mid n-1)\right),
\end{gathered}
$$

$\hat{y}_{n, I}^{\prime}(n)$ and $\hat{y}_{n, Q}^{\prime}(n)$ are updated when $x_{n, I}(n)$ and $x_{n, Q}(n)$ become available; the amounts to modify the $\hat{y}_{n, I}^{\prime}(n \mid n-1)$ and $\hat{y}_{n, Q}^{\prime}(n \mid n-1)$, i.e., former estimates by a quantity proportional to the innovative part of the new observation.

The updating stage of the Kalman filter includes an innovative process which is one of the essential concepts in the Kalman filter technique. The innovative approach can be described as:

$$
\begin{gathered}
\hat{y}_{n, I}^{\prime}(n \mid n-1)=x_{n, I}(n)-H_{I}(n) \hat{y}_{n, I}^{\prime}(n \mid n-1), \\
\hat{y}_{n, Q}^{\prime}(n \mid n-1)=x_{n, Q}(n)-H_{Q}(n) \hat{y}_{n, Q}^{\prime}(n \mid n-1),
\end{gathered}
$$

The estimation error can be explained by the equation below, when $x_{n, I}(n)$ and $x_{n, Q}(n)$ is estimated by $\hat{y}_{n, I}^{\prime}(n)$ and $\hat{y}_{n, Q}^{\prime}(n)$.

$$
\begin{aligned}
e_{I}(n) & =\hat{y}_{n, I}^{\prime}(n)-x_{n, I}(n), \\
e_{Q}(n) & =\hat{y}_{n, Q}^{\prime}(n)-x_{n, Q}(n),
\end{aligned}
$$

\section{Results and Discussion}

The performance analysis of the assumed FD DC transceiver model with its nonidealities and their linearization techniques, analog/RF SI cancellation, and digital residual SI cancellation technique is simulated and verified explicitly with the help of well-known simulator "MATLAB/Simulink". OFDM is used as a baseband test signal for simulating a realistic signal. Non-idealities simulated with their linearization techniques are IQ mixer imbalance and PA nonlinearity in the Tx chain and LNA linearity and IQ mixer imbalance in the Rx chain. The mismatch between the I and $Q$ branch of the IQ mixer is adjusted such that the required IRR can be achieved. PA nonlinearity is modeled with the help of a non-linear memory polynomial, and LNA nonlinearity with a quasi-memoryless model. Other components such as VGA, DAC, and ADC are considered linear. VGA is considered linear with complex gain and ADC and DAC as uniform quantization processes.

Two separate antennae are used for transmission and reception, and the antenna separation for isolation is considered and modeled as path loss of: (i) $30 \mathrm{~dB}$ and (ii) $40 \mathrm{~dB}$. The multi-path channel between transmit and receive antenna is static for SI signal and is modeled, such that the line of sight (LOS) path and two non-line of sight (NLOS) paths of the multi-path channel have an average power ratio of $35 \mathrm{~dB}$. However, the rest of the channel is dynamic in nature, i.e., due to motion of surrounding things such as humans, vehicles or other voices, and is modeled as noise. Noise is modeled as AWGN with the power of $-174 \mathrm{dBm} / \mathrm{Hz}$, mean zero and variance $\sigma_{W}^{2}$. Parameters of test signal and system are given in Tables 1 and 2. Table 3 shows the non-linear and linear parameters of components of the Tx and Rx chain. 
Table 1. Test signal "OFDM" parameters.

\begin{tabular}{cc}
\hline Parameters & Values \\
\hline Modulation Scheme & 16 QAM \\
\hline Number of Sub-carriers & 64 \\
\hline Number of Data sub-carriers & 48 \\
\hline Symbol Time & $3.2 \mu \mathrm{s}$ \\
\hline Guard Interval or CP Duration & $25 \%$ of symbol length \\
\hline
\end{tabular}

Table 2. System parameters.

\begin{tabular}{cc}
\hline Parameters & Values \\
\hline Bandwidth & $20 \mathrm{MHz}$ \\
\hline Receiver thermal noise floor & $-103.0 \mathrm{dBm}$ \\
\hline Sensitivity & $-88.9 \mathrm{dBm}$ \\
\hline Receiver noise figure & $4.1 \mathrm{~dB}$ \\
\hline Noise power & $-174 \mathrm{dBm} / \mathrm{Hz}$ \\
\hline Power at receiver & $-88.9 \mathrm{dBm}$ \\
\hline Transmit power & 5 to $40 \mathrm{dBm}$ \\
\hline Antenna separation & $30 \mathrm{~dB}$ and $40 \mathrm{~dB}$ \\
\hline Carrier frequency & $2 \mathrm{GHz}$ \\
\hline
\end{tabular}

Table 3. Non-linear and linear parameters of transmitter and receiver chain.

\begin{tabular}{ccccc}
\hline Component & $\begin{array}{c}\text { Gain } \\
(\mathbf{d B})\end{array}$ & $\begin{array}{c}\text { IIP2 } \\
(\mathbf{d B m})\end{array}$ & $\begin{array}{c}\text { IIP3 } \\
(\mathbf{d B m})\end{array}$ & $\begin{array}{c}\text { NF } \\
(\mathbf{d B})\end{array}$ \\
\hline IQ Mixer (Tx and Rx) & 6 & 50 & 15 & 4 \\
\hline PA & 27 & - & 20 & 5 \\
\hline LNA & 25 & - & 5 & 4.1 \\
\hline VGA & $1-51$ & 50 & 20 & 4 \\
\hline $\begin{array}{c}\text { IQ Mixer IRR (dB) } \\
(\text { Tx and Rx) }\end{array}$ & 13 & - & - & - \\
\hline PA memory length & 6 & - & - & - \\
\hline & Bits & 12 & - & - \\
\cline { 2 - 5 } ADC & P-P voltage range & $4.5 \mathrm{~V}$ & - & - \\
\cline { 2 - 5 } & PAPR & $10 \mathrm{~dB}$ & & - \\
\hline
\end{tabular}

The efficiency and performance of the FD transceiver are calculated based on link budget analysis, thereby computing powers of different components of SI signal and SINR.

\subsection{Link Budget Analysis}

Link budget analysis describes the details of signal components at every stage of Tx and Rx. These details include noise figure, gain, SNR, and IIP3, as shown in Figure 8. 


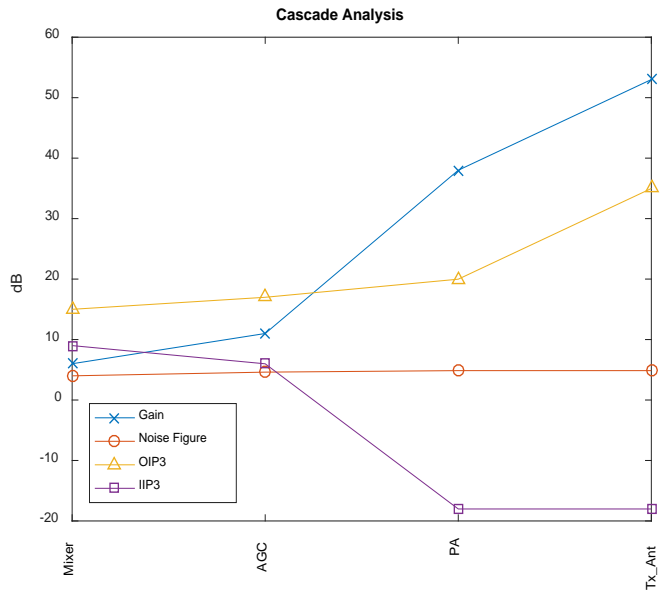

(a)

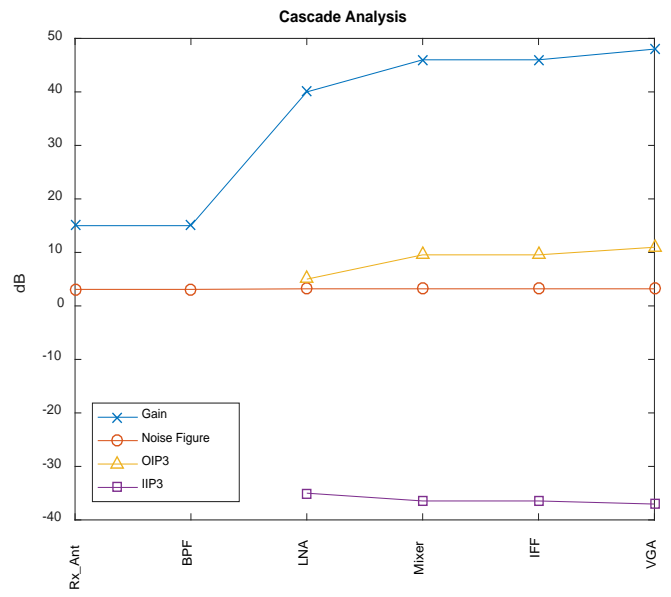

(b)

Figure 8. Link budget analysis: (a) Transmitter chain; (b) receiver chain.

\subsection{Power Level of Different Components of SI. Signal After RF/Analog SI Cancellation}

The power of different components of the SI signal at Rx input without any SI cancellation is shown in Figure $9 \mathrm{a} . P_{S I}$ and $P_{S I, i m}$ are much stronger signal components and have much higher powers compared to the desired signal, which has a power of approximately $-55 \mathrm{dBm}$ at an Rx sensitivity level of $-88.9 \mathrm{dBm}$. At Tx power of $5 \mathrm{dBm}, P_{S I}$ is $\sim-15 \mathrm{dBm}$ and $P_{S I, i m}$ I s $\sim 40 \mathrm{dBm}$, hence $P_{S I}$ is $\sim 40 \mathrm{dBm}$ and $P_{S I, i m}$ is $\sim 15 \mathrm{dBm}$ stronger than the desired signal, and $P_{S I}$ is $\sim 73.9 \mathrm{dBm}$ and $P_{S I, i m}$ is $\sim 48.9 \mathrm{dBm}$ stronger than the Rx sensitivity. However, $P_{I M D}$ and $P_{I M D, i m}$ are not very strong but are still higher than the sensitivity level of Rx at higher transmit powers, i.e., above $25 \mathrm{dBm}$. Figure $9 \mathrm{~b}, \mathrm{c}$ shows the results of the proposed SI cancellation technique, i.e., CSI with LS estimator. With this technique, along with $30 \mathrm{~dB}$ antenna isolation as shown in Figure $9 \mathrm{~b}$, the power of all the SI signal components drops below the desired signal and Rx sensitivity level, except the image component $P_{S I, i m}$.

Hence, now the only SI component with a power level above the Rx sensitivity is $P_{S I, i m}$. It can be observed in Figure $9 \mathrm{c}$ that by using the CSI technique along with $40 \mathrm{~dB}$ antenna isolation, $P_{S I, i m}$ power also falls below Rx sensitivity. For antenna isolation of $30 \mathrm{~dB}$ with CSI, $P_{S I, i m}$ remains below the desired signal power level for transmit power levels higher than $20 \mathrm{dBm}$. Similarly, for antenna isolation of $40 \mathrm{~dB}$ with CSI, $P_{S I, i m}$ remains below the desired signal power level for transmit power levels higher than $30 \mathrm{dBm}$. The receiver can also be protected from high power SI signal by using only a single stage of a Kautz and GALL filter. Figure 9d,e shows that after passing through the Kautz and GALL filter, SI signal power is reduced to a much lower level that is below sensitivity level, and the power received at the input of the $\mathrm{Rx}$ is approximately equal to the desired signal, which shows that SI signal is almost cancelled, and the remaining error can be treated as low power noise. From Figure 9, SI signal power is significantly dominant over the desired signal before applying the proposed SI cancellation techniques. As a result, the total power at the Rx input is approximately equal to the SI signal power. However, after applying SI cancellation techniques, the SI power becomes negligibly small, and the total power is predominantly similar to the signal power. 


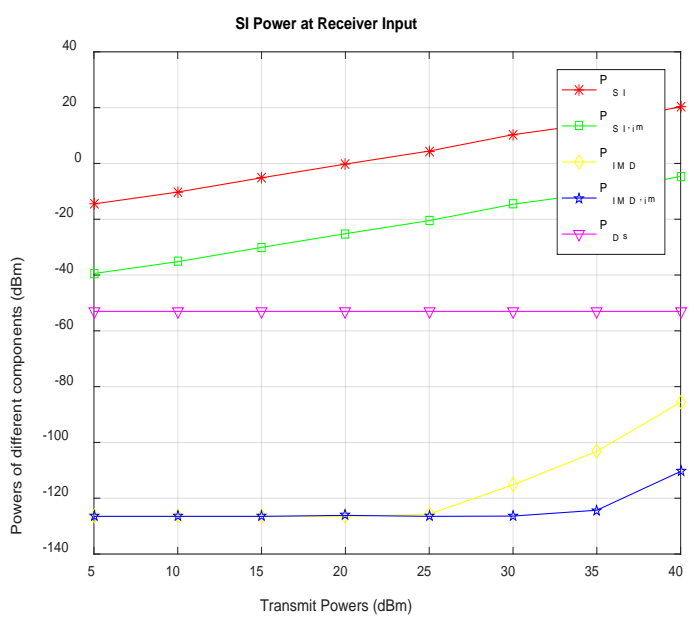

(a)

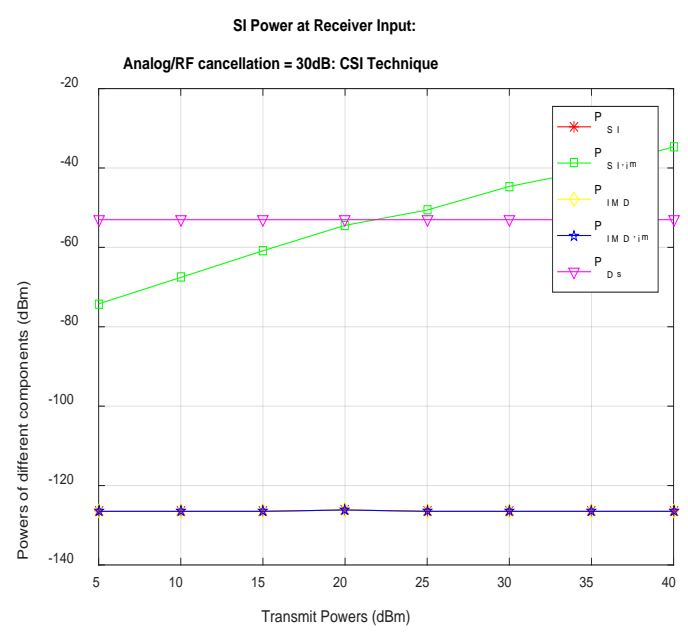

(b)

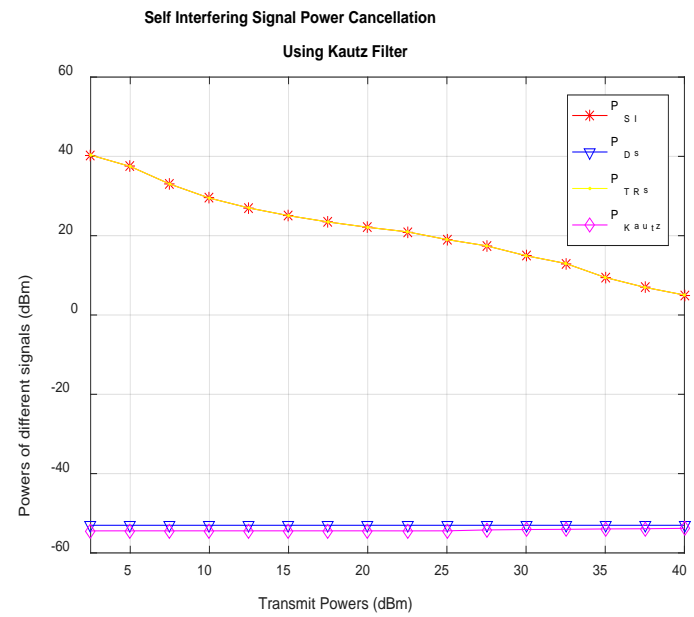

(d)

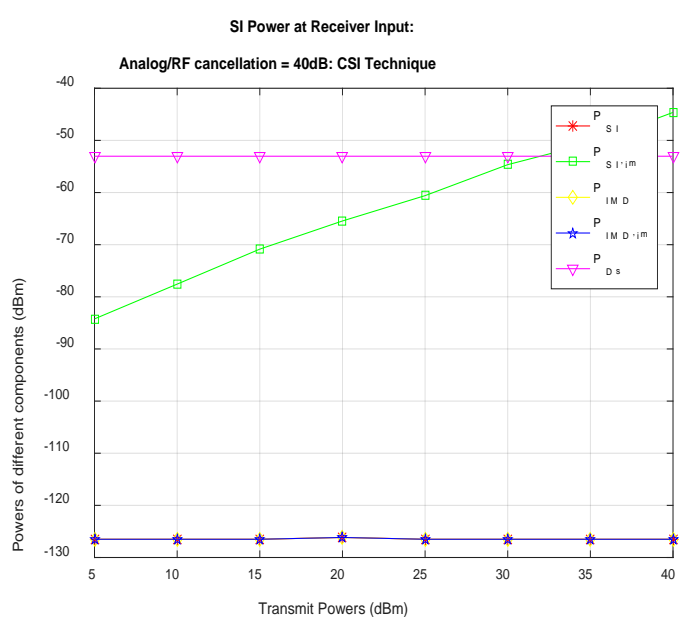

(c)

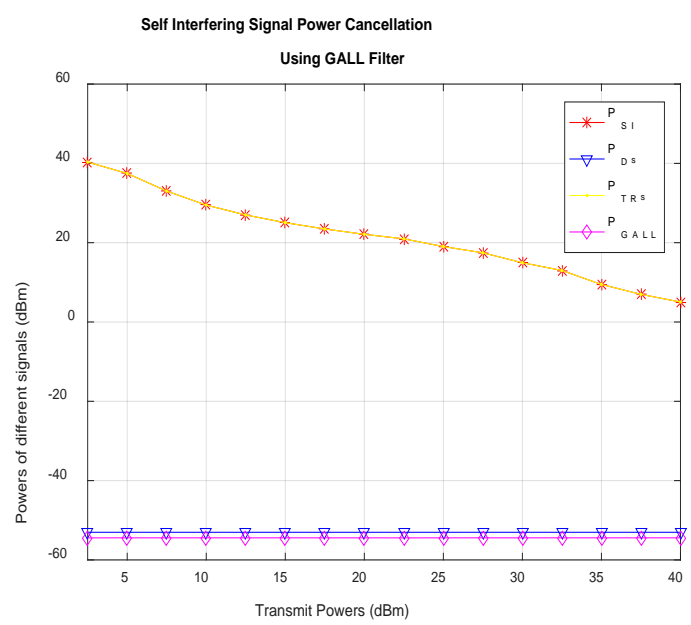

(e)

Figure 9. Power of self-interfering signal components before and after SI cancellation techniques. SI power at receiver: (a) without any SI cancellation; (b) with $30 \mathrm{~dB}$ antenna isolation and CSI technique; (c) with $40 \mathrm{~dB}$ antenna isolation and CSI technique; (d) with the Kautz filter as an SI cancellation technique; (e) with the GALL filter as an SI cancellation technique. 


\section{3. $\operatorname{SINR}$}

Figure 10a shows SINR after the implementation of analog SI cancellation techniques. Kautz and GALL filters are better than CSI techniques, because for Kautz and GALL filters, although separate antennae are used, no antenna cancellation techniques are required. Figure 10b shows the SINR before digital signal processing and after implementing the digital SI cancellation technique, i.e., the Kalman filter. By applying the Kalman filter on the digital side, the system SINR improved to a significant value, i.e., approximately equal to $S I N R_{\text {Ideal }}$, which falls because of the presence of the residual SI signal.

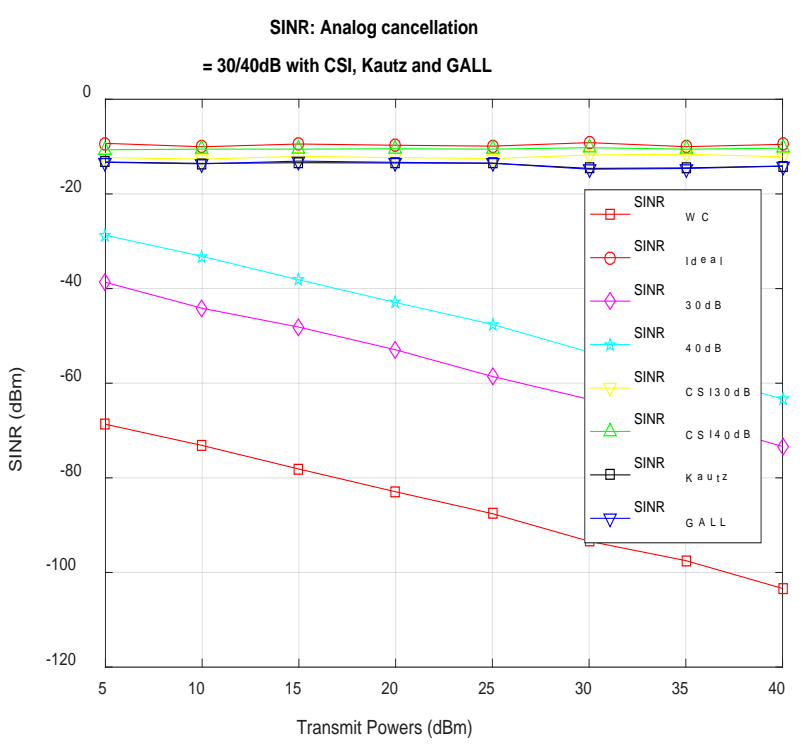

(a)

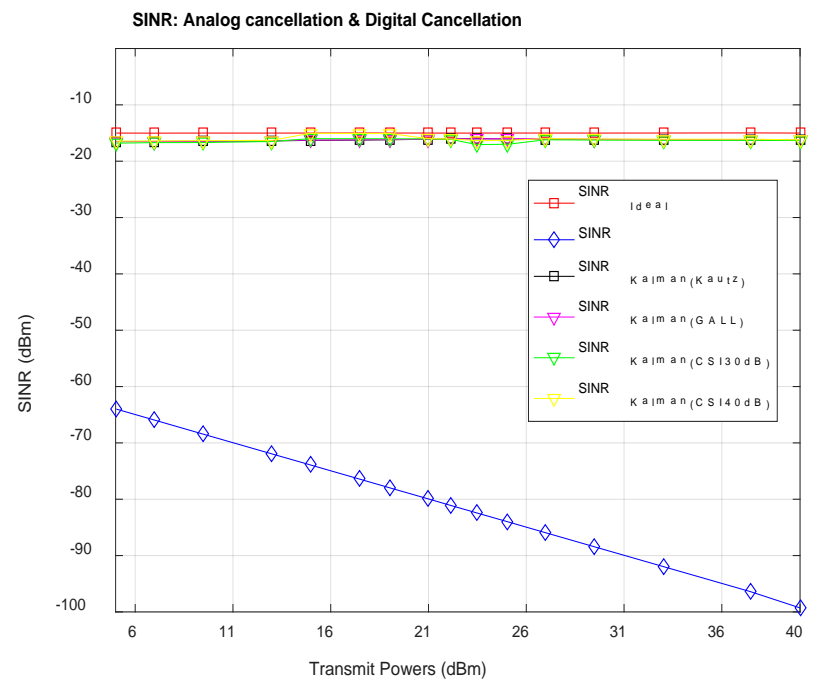

(b)

Figure 10. SINR using analog and digital cancellation techniques. (a) SINR after analog SI cancellation $30 \mathrm{~dB}$ and $40 \mathrm{~dB}$ antenna isolation with CSI technique, Kautz and GALL filter; (b) SINR after both analog and digital SI cancellation.

\subsection{Linearization}

A performance analysis of the FD transceiver's Tx chain components is carried out separately, and results are shown through constellation diagrams and spectrum analyzers.

Figure 11a-c shows the signal trajectory of the ideal, imbalance, and compensated IQ mixer of the Tx chain, respectively. Figure 11c shows that the compensated IQ mixer follows nearly the same signal trajectory as that of an ideal IQ mixer (Figure 11a). Figure 11d-f shows the constellation diagram of the ideal, imbalance, and compensated IQ mixer of the TX chain, respectively. It is clear from the constellation diagram Figure $11 \mathrm{f}$ that the compensated results are approximately nearer to those of the ideal IQ mixer (Figure 11d). The IQ mixer in the Tx chain is tested separately with its linearization techniques before putting it into the transceiver design, and then the combined results are evaluated. The signal used to test the IQ mixer model is a simple 16-quadrature amplitude modulation (QAM) signal.

PA linearity analysis is carried out by examining PA PSD and its input to output relationship. Figure 12 shows the output vs. input relationship of PA with and without linearization techniques. The non-linear amplifier shows great diversity due to intermodulation products of the third order. However, after applying linearization techniques, the output is the linear product of input.

Figure 13a,b shows the results of PA before and after applying linearization techniques. It is clear from the figures that inter-modulation products have approximately equal power spectral density as linear gain and, hence, result in large signal distortion. In contrast, linearization techniques help with insufficient reduction of spectral regrowth and reduce PA intermodulation product. However, the results obtained from PD linearization are better than those received by applying the FF linearization technique. The downside of PD 
linearization techniques is that they have computational complexities, but they provide better results.

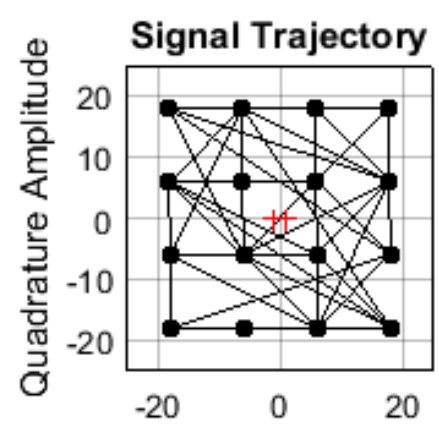

In-phase Amplitude

(a)

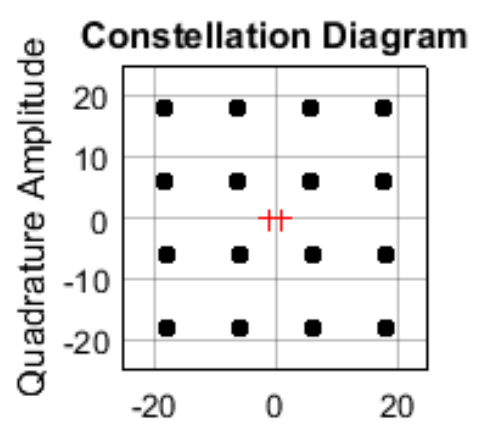

In-phase Amplitude

(d)

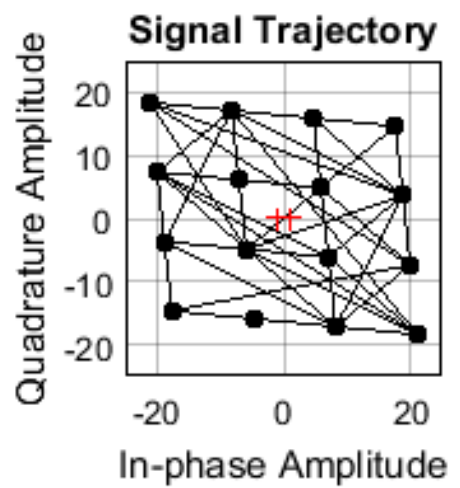

(b)

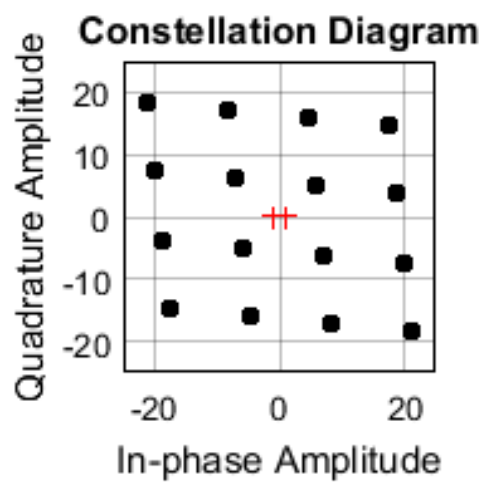

(e)

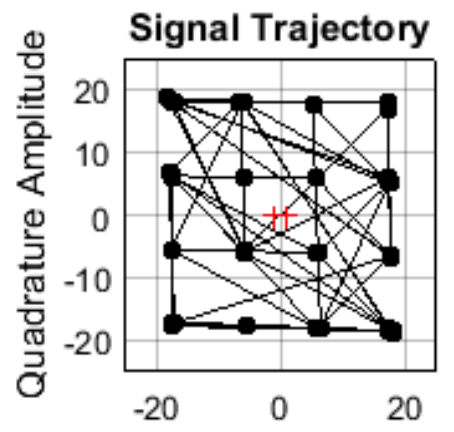

In-phase Amplitude

(c)

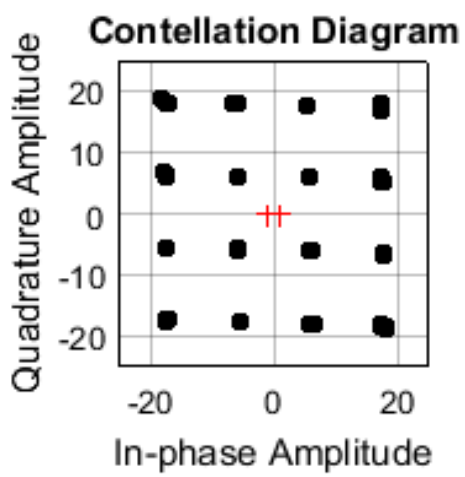

(f)

Figure 11. The signal trajectory and the constellation diagram of IQ mixer of transmitter chain with $25 \%$ gain and $25^{\circ}$ phase imbalance. $(\mathbf{a}-\mathbf{c})$ show the signal trajectory of the ideal, imbalance, and compensated IQ mixer of the Tx chain, respectively. (d-f) show the constellation diagram of the ideal, imbalance, and compensated IQ mixer of the TX chain, respectively.

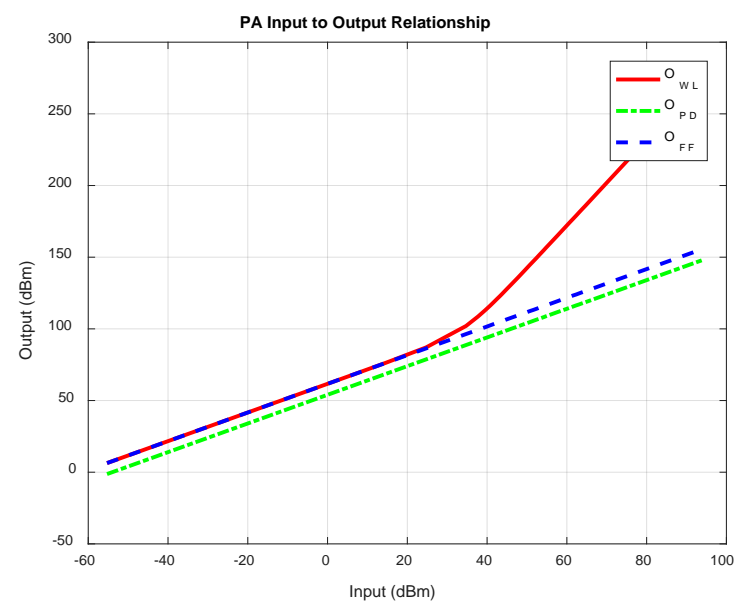

Figure 12. Output vs. input relationship of PA with and without linearization techniques. 


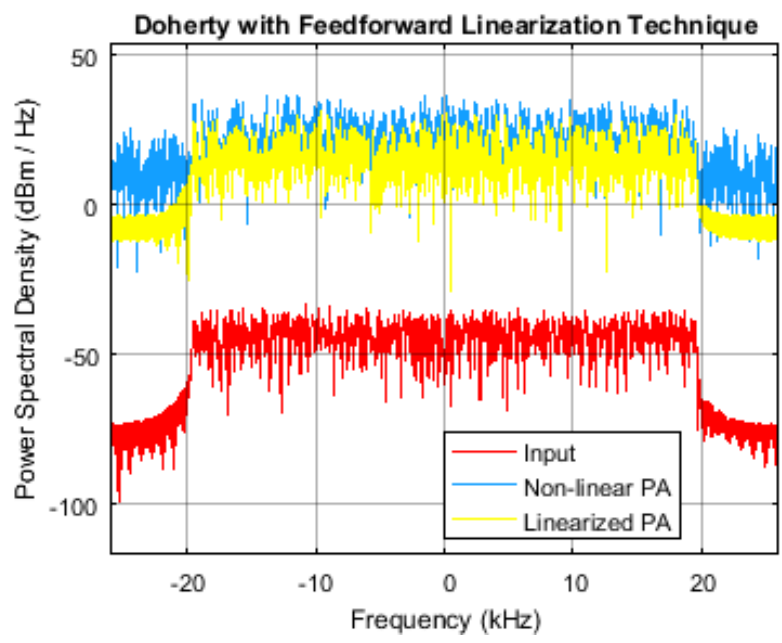

(a)

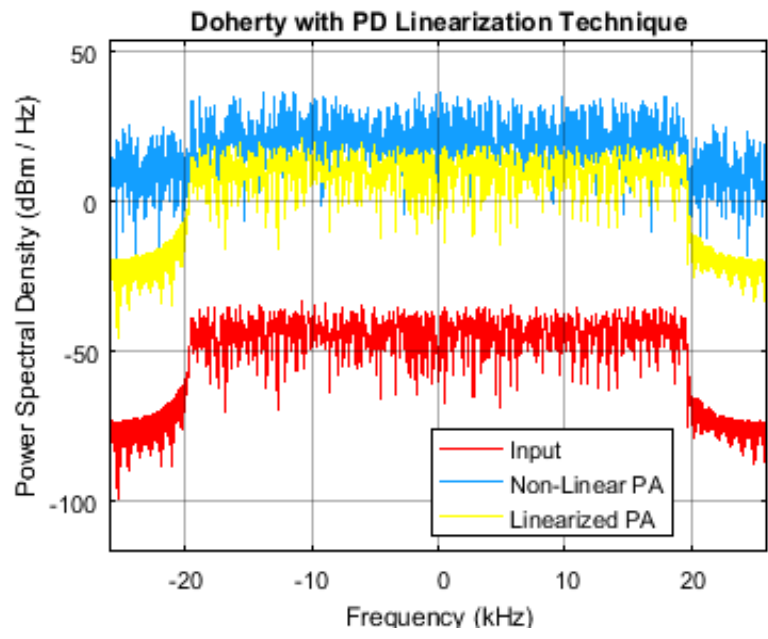

(b)

Figure 13. Doherty amplifier technique with feedforward linearization technique (a); and pre-distortion linearization technique (b).

Unlike the transmitter chain, receiver chain performance analysis is carried out using the metric BER vs. SNR. LNA linearity analysis is performed separately and examined through spectral power density and output to input relationship of LNA with and without linearization techniques. Figure 14 shows the relationship between LNA output and input. Non-linear LNA output shows non-linear behavior as input increases above $0 \mathrm{dBm}$ and offers a sizeable non-linear behavior. However, after applying linearization techniques, the output and input relationship become linear, and output increases linearly with the increase of input. Figure 15 shows the PSD of LNA. Linearization techniques help in a reduced amount of spectral regrowth and decrease the intermodulation product power spectral density.

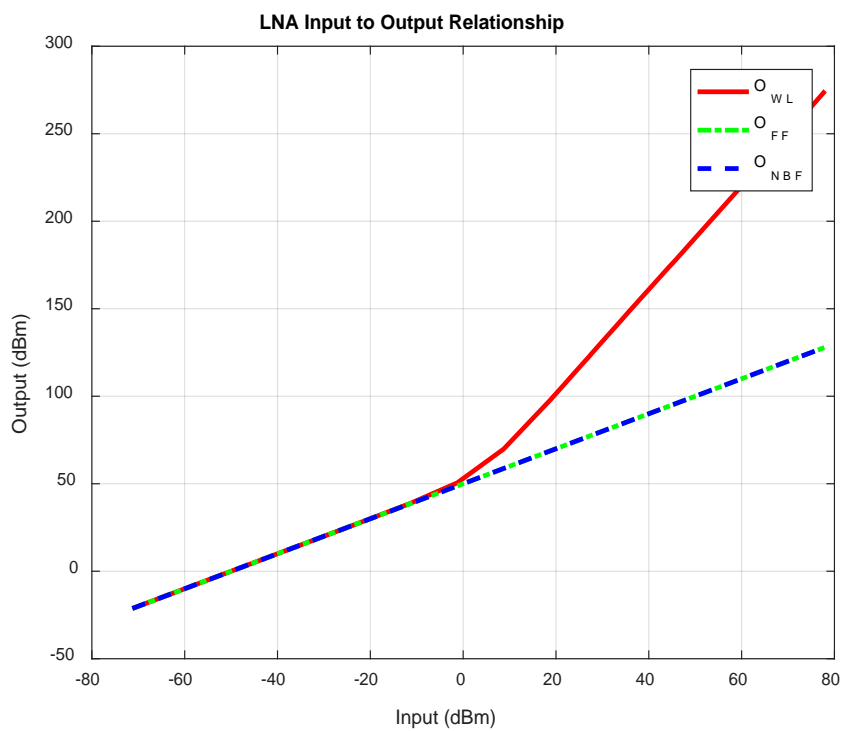

Figure 14. Output vs. input relationship of LNA with and without linearization techniques. 


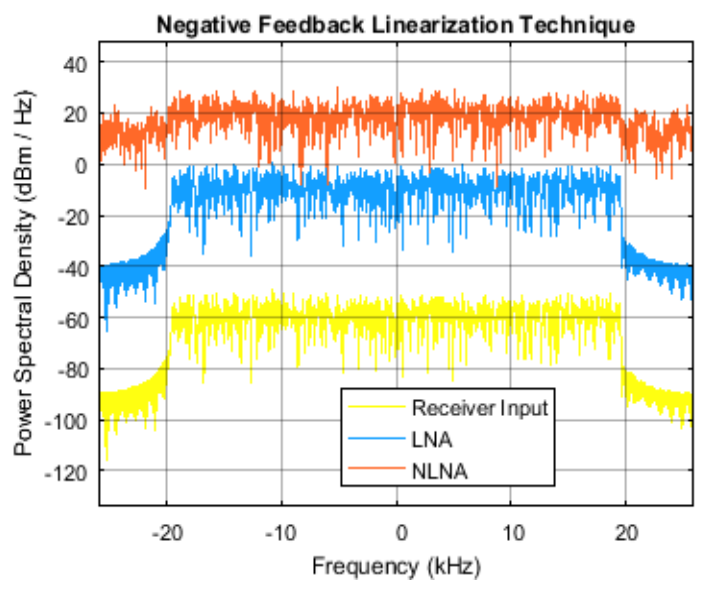

(a)

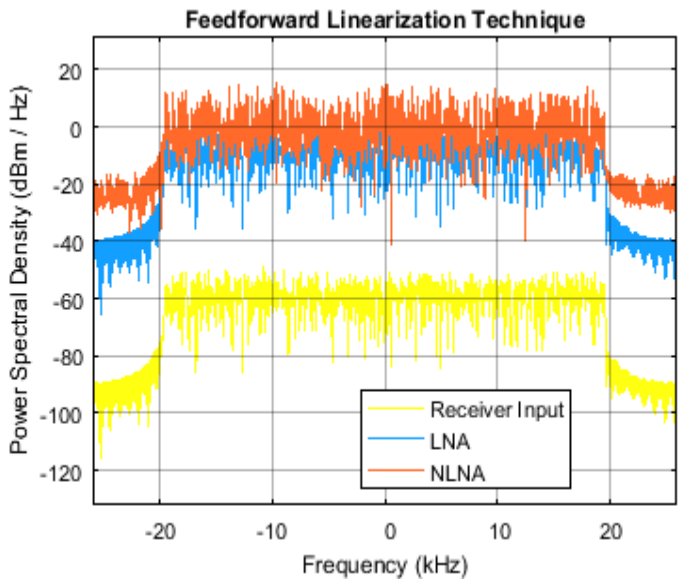

(b)

Figure 15. Non-linear LNA with negative feedback linearization technique (a); and feedforward linearization technique (b).

After the linearization of LNA and IQ mixer, Rx performance is carried out, and BER is calculated before and after the linearization of Rx front-end components. Figure 16 shows the curve of Rx BER vs. SNR. Due to linearization techniques, improved BER is achieved. However, in the presence of Rx impairments, BER offers quite strange behavior, and up to $20 \mathrm{~dB}$ SNR, BER is relatively high, i.e., even below $10^{-1}$.

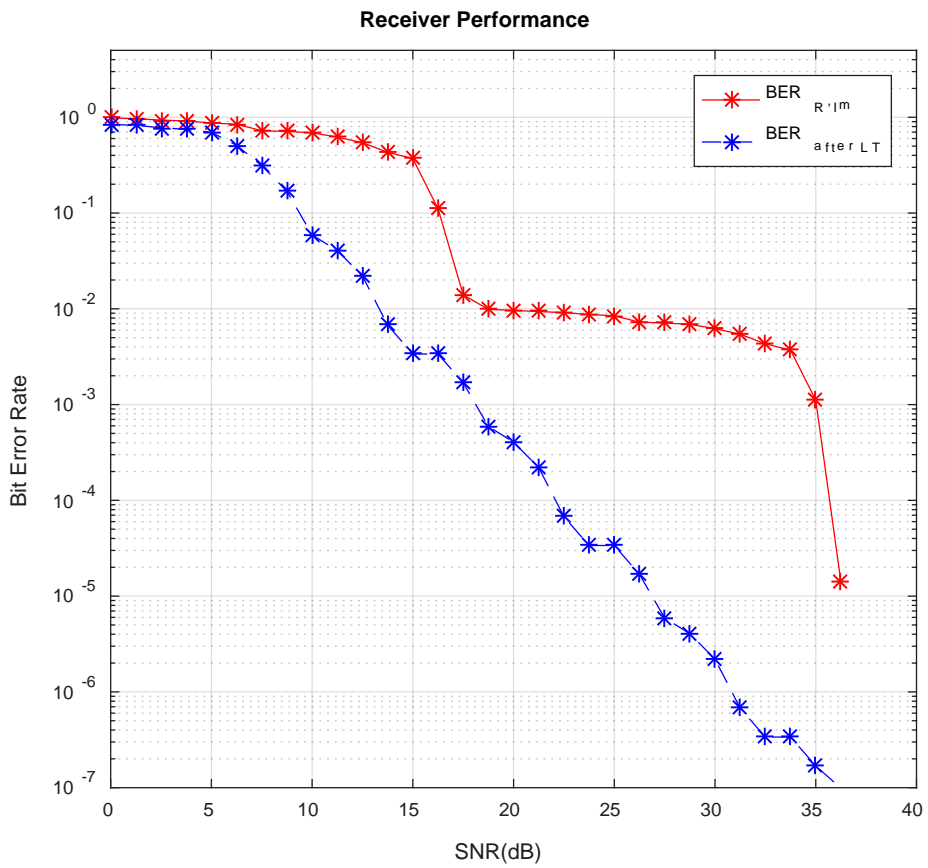

Figure 16. Receiver performance; BER vs. SNR.

Comparison between proposed techniques for self-interference cancellation with work previously carried out in the field of FD communication systems is presented in Table 4 . 
Table 4. Comparison of proposed work with previous works.

\begin{tabular}{|c|c|c|c|c|c|}
\hline \multicolumn{3}{|c|}{ Techniques } & \multicolumn{2}{|c|}{ Results } & \multirow[b]{2}{*}{$\begin{array}{c}\text { Reference } \\
\text { Papers }\end{array}$} \\
\hline Analog/RF SIC & Digital SIC & $\begin{array}{c}\text { Linearization } \\
\text { Techniques (LT) }\end{array}$ & Analog/RF SIC & Digital SIC & \\
\hline $\begin{array}{c}\text { Auxiliary path } \\
\text { (consisting of DAC, IQ, } \\
\text { PA and RF attenuator) }\end{array}$ & Gain factor & - & \multicolumn{2}{|c|}{$74 \mathrm{~dB}$} & [17] \\
\hline \multirow[t]{2}{*}{$\begin{array}{l}\text { Robust algorithm } \\
\text { known as SINC } \\
\text { interpolation }\end{array}$} & \multirow[t]{2}{*}{ LS algorithm } & \multirow[t]{2}{*}{ - } & $60 \mathrm{~dB}$ & $\begin{array}{c}\text { Residual linear } \\
\text { components by } \\
50 \mathrm{~dB} \\
\text { Non-linear } \\
\text { components by } \\
20 \mathrm{~dB} \text {. }\end{array}$ & \multirow[t]{2}{*}[74]{} \\
\hline & & & \multicolumn{2}{|c|}{$\begin{array}{c}\text { Hybrid model enables SI } \\
\text { cancellation near to receiver noise } \\
\text { floor up to } 110 \mathrm{~dB}\end{array}$} & \\
\hline \multirow{2}{*}{$\begin{array}{l}\text { One tap filter depicting } \\
\text { the delay, phase and } \\
\text { attenuation of main } \\
\text { coupling path }\end{array}$} & \multirow[b]{2}{*}{ LS estimator } & \multirow[b]{2}{*}{-} & \multirow[b]{2}{*}{$30 \mathrm{~dB} \& 20 \mathrm{~dB}$} & $\begin{array}{l}\text { Linear Model: } \\
25 \mathrm{~dB}\end{array}$ & \multirow[b]{2}{*}{ [39] } \\
\hline & & & & $\begin{array}{l}\text { Widely Linear } \\
\text { Model: } 35 \text { or } \\
50 \mathrm{~dB}\end{array}$ & \\
\hline \multirow{2}{*}{$\begin{array}{l}\text { Passive isolation } \\
\text { (special antenna } \\
\text { design) }\end{array}$} & \multirow{2}{*}{-} & \multirow[t]{2}{*}{-} & $\begin{array}{l}\text { Passive } \\
\text { isolation: } \\
60-70 \mathrm{~dB}\end{array}$ & $\mathrm{C}_{2}$ & \multirow{2}{*}{ [75] } \\
\hline & & & \multicolumn{2}{|c|}{$\begin{array}{l}100 \mathrm{~dB} \text { of overall self-interference } \\
\text { suppression }\end{array}$} & \\
\hline \multirow{2}{*}{$\begin{array}{l}\text { Presents a novel RF } \\
\text { circuit architecture }\end{array}$} & \multirow{2}{*}{-} & \multirow[t]{2}{*}{-} & $\begin{array}{c}100 \mathrm{MHz} \\
\text { waveform } \\
\text { bandwidth: } \\
41 \mathrm{~dB} \text { total } \\
\text { cancellation }\end{array}$ & \multirow[t]{2}{*}{-} & \multirow{2}{*}{ [76] } \\
\hline & & & $\begin{array}{l}20 \mathrm{MHz} \text { carrier } \\
\text { bandwidth: } \\
60 \mathrm{~dB} \text { total } \\
\text { cancellation }\end{array}$ & & \\
\hline $\begin{array}{l}\text { Digitally controlled RF } \\
\text { self-interference } \\
\text { canceller structure }\end{array}$ & - & - & $\begin{array}{c}\text { More than } \\
40 \text { dBs of active } \\
\text { RF cancellation } \\
\text { gain up to } \\
80 \mathrm{MHz} \\
\text { instantaneous } \\
\text { waveform } \\
\text { bandwidths }\end{array}$ & - & [77] \\
\hline $\begin{array}{l}\text { Used a shared antenna } \\
\text { and a circulator, an } \\
\text { adjustable impedance } \\
\text { mismatch terminal } \\
\text { (IMT) circuit at the } \\
\text { antenna interface is } \\
\text { added for cancellation } \\
\text { of SI }\end{array}$ & - & - & $40 \mathrm{~dB}$ & - & [78] \\
\hline $\begin{array}{l}\text { Block-adaptive } \\
\text { Learning algorithm } \\
\text { through decorrelation } \\
\text { for RF cancellation }\end{array}$ & - & - & $54 \mathrm{~dB}$ & - & [79] \\
\hline
\end{tabular}


Table 4. Cont

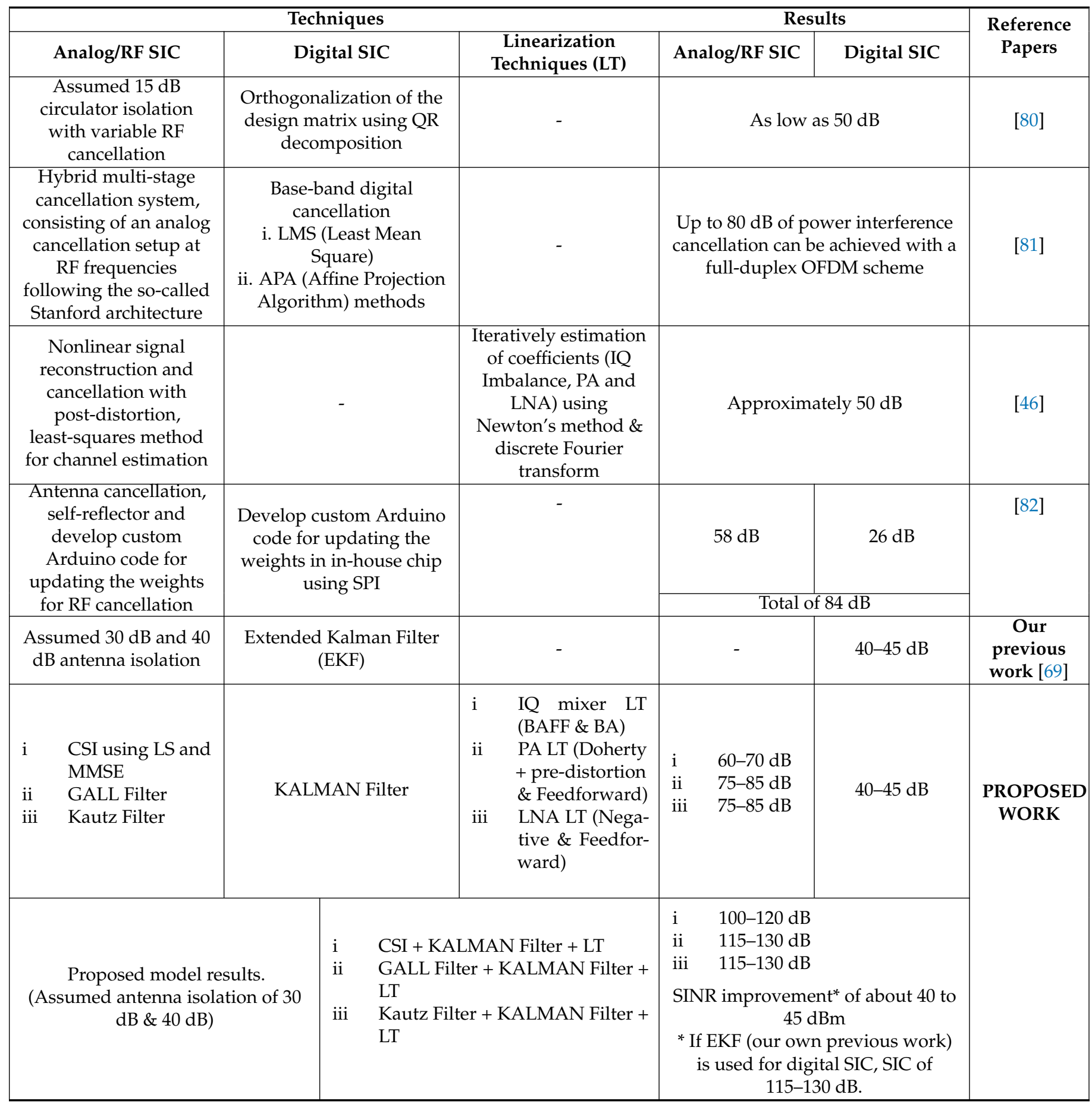

\section{Conclusions}

FD has gained a great deal of attention with regard to next-generation wireless radio communication systems such as 5G. Recently, the attention of researchers in FD wireless radio communication systems has led to the study of SI cancellation techniques for colocated Tx and Rx pairs. For efficient and reliable FD systems, SI needs to be mitigated to a sufficiently low level, i.e., below the Rx sensitivity and noise floor both on the analog and the digital side. Even after applying RF/analog cancellation techniques, there is still residual SI signal. Digital SI cancellation techniques are applied to mitigate the residual SI signal. In this article, two SI cancellation techniques, a digital SI cancellation technique, and 
different techniques for reducing the nonlinearity are presented. System performance was evaluated based on SINR, the power of different components of SI signal, and link budget analysis. Through the CSI active cancellation technique employed in the LS estimator, the SI signal cancellation up to $70 \mathrm{~dB}$ was achieved. SINR obtained, in this case, is almost equal to the ideal, i.e., the one calculated at the Rx input. The Kautz and GALL filters are adaptive filters used to cancel the SI signal at the Rx side, and they achieve 70-75 $\mathrm{dB}$ and $80-85 \mathrm{~dB}$ SI cancellation, respectively. The Kalman filter is applied to mitigate the residual SI signal on the digital side, and the SI cancellation of about 30 to $35 \mathrm{~dB}$ is achieved. SINR obtained at the Rx side after applying the Kalman filter is almost equal to the ideal, i.e., the one calculated at the Rx output, i.e., before digital signal processing. Hence, the SI signal mitigation achieved after both RF/analog and digital SI cancellation techniques is approximately up to 100 to $130 \mathrm{~dB}$.

Author Contributions: Conceptualization, A.A. and S.M.C.; data curation, A.A. and M.R.; formal analysis, A.A. and S.M.C.; funding acquisition, M.R. and A.H.; investigation, A.A. and A.H.; methodology, A.A. and S.M.C.; resources, M.R. and A.H.; writing-original draft preparation, A.A.; writing-review and editing, A.A. and A.H.; supervision, A.H. and S.M.C. All authors have read and agreed to the published version of the manuscript.

Funding: This research received no external funding.

Institutional Review Board Statement: Not applicable.

Informed Consent Statement: Not applicable.

Data Availability Statement: Not applicable.

Conflicts of Interest: The authors declare no conflict of interest.

\section{Nomenclature}

$P_{S I}$

$P_{S I, i m}$

$P_{I M D}$

$P_{I M D, i m}$

$P_{D s}$

$P_{T R S}$

$P_{\text {Kautz }}$

$P_{\text {GALL }}$

SINR and SINR $W C$

$S_{I N R} 30 d B$

$S_{I N R} 40 d B$

$S I N R_{\text {CSI_30dB }}$

SINR CSI_40dB

SINR Kautz

$S_{I N R_{G A L L}}$

$\operatorname{SINR}_{\text {Kalman (Kautz) }}$

SINR Kalman(GALL)

$\operatorname{SINR}_{\text {Kalman (CSI30dB) }}$

$\operatorname{SINR}_{\text {Kalman }(C S I 40 d B)}$

$O_{W L}$

$O_{P D}$

$O_{F F}$

$O_{N F B}$

$B E R_{R, I m}$

$B E R_{\text {After } L T}$
Power of direct component

Power of image component

Power of intermediate distortion component

Power of image component of IMD

Power of desired Signal

Total power at the receiver input

Power after applying analog/RF cancellation using Kautz filter

Power after applying analog/RF cancellation using GALL filter

SINR without any cancellation technique

SINR with $30 \mathrm{~dB}$ of antenna cancellation

SINR with $40 \mathrm{~dB}$ of antenna cancellation

SINR with CSI and $30 \mathrm{~dB}$ antenna cancellation

SINR with CSI and $40 \mathrm{~dB}$ antenna cancellation

SINR after Kautz filter implementation

SINR after GALL filter implementation

SINR after Kautz filter with Kalman filter implementation

SINR after GALL filter with Kalman filter implementation

SINR after CSI and $30 \mathrm{~dB}$ antenna cancellation with Kalman filter implementation

SINR after CSI and $40 \mathrm{~dB}$ antenna cancellation with Kalman filter implementation

The output of a non-linear amplifier

The output of PA after PD technique

The output of PA after FF technique

The output of PA after NFB technique

$B E R$ in the presence of receiver front end impairments

BER after applying linearization techniques to receiver front end components 


\section{References}

1. Choi, J.I.; Jain, M.; Srinivasan, K.; Levis, P.; Katti, S. Achieving Single Channel, Full Duplex Wireless Communication. In Proceedings of the Sixteenth Annual International Conference on Mobile Computing and Networking (MobiCom'10), Houston, TX, USA, 12-14 November 2019; Association for Computing Machinery: New York, NY, USA, 2010; pp. 1-12. [CrossRef]

2. Weeraddana, P.C.; Codreanu, M.; Latvaaho, M.; Ephremides, A. On the effect of self-interference cancelation in multi-hop wireless networks. EURASIP J. Wirel. Commun. Netw. 2010, 2010, 1-10. [CrossRef]

3. Korpi, D.; Riihonen, T.; Syrjala, V.; Anttila, L.; Valkama, M.; Wichman, R. Full-duplex transceiver system calculations: Analysis of ADC and linearity challenges. IEEE Trans. Wirel. Commun. 2014, 13, 3821-3836. [CrossRef]

4. Knox, M.E. Single antenna full-duplex communications using a common carrier. In Proceedings of the WAMICON 2012 IEEE Wireless and Microwave Technology Conference, Cocoa Beach, FL, USA, 15-17 April 2012; pp. 1-6.

5. Cox, C.; Ackerman, E. Demonstration of a single-aperture, full-duplex communication system. In Proceedings of the 2013 IEEE Radio and Wireless Symposium, Austin, TX, USA, 20-23 January 2013; pp. 148-150.

6. Jain, M.; Choi, J.I.; Kim, T.; Bharadia, D.; Seth, S.; Srinivasan, K.; Levis, P.; Katti, S.; Sinha, P. Practical, real-time, full-duplex wireless. In Proceedings of the 17th Annual International Conference on Mobile Computing and Networking, Las Vegas, NV, USA, 19-23 September 2011; pp. 301-312.

7. Sahai, A.; Patel, G.; Sabharwal, A. Pushing the limits of full-duplex: Design and real-time implementation. arXiv 2011, arXiv:1107.0607.

8. Duarte, M.; Sabharwal, A. Full-duplex wireless communications using off-the-shelf radios: Feasibility and first results. In Proceedings of the 2010 Conference Record of the Forty-Fourth Asilomar Conference on Signals, Systems, and Computers, Pacific Grove, CA, USA, 7-10 November 2010; pp. 1558-1562.

9. Phunganmgern, N.; Uthansakul, P.; Uthansakul, M. Digital and RF interference cancellation for single-channel full-duplex transceiver using a single antenna. In Proceedings of the 2013 10th International Conference on Electrical Engineering/Electronics, Computer, Telecommunications, and Information Technology, Krabi, Thailand, 15-17 May 2013; pp. 1-5.

10. Anderson, C.R.; Krislmamoorthy, S.; Ran Son, C.G.; Lemon, T.J.; Newhall, W.G.; Kummetz, T.; Reed, J.H. Antenna isolation, wideband multi-path propagation measurements, and interference mitigation for on-frequency repeaters. In Proceedings of the IEEE Southeast Conference, Greensboro, NC, USA, 26-29 March 2004; pp. 110-114.

11. Carroll, A.; Heiser, G. An analysis of power consumption in a smartphone. In Proceedings of the USENIX Annual Technical Conference, Boston, MA, USA, 23-25 June 2010; p. 21.

12. Singh, V.; Gadre, A.; Kumar, S. Full Duplex Radios: Are we there yet? In Proceedings of the 19th ACM Workshop on Hot Topics in Networks, Virtual Event, New York, NY, USA, 4-6 November 2020; pp. 117-124.

13. Nguyen, B.C.; Thang, N.N.; Tran, X.M.; Dung, L.T. Impacts of imperfect channel state information, transceiver hardware, and self-interference cancellation on the performance of full-duplex mimo relay system. Sensors 2020, 20, 1671. [CrossRef] [PubMed]

14. Alves, H.; Riihonen, T.; Suraweera, H.A. Full-Duplex Communications for Future Wireless Networks; Springer: Singapore, 2020.

15. Sahai, A.; Patel, G.; Sabharwal, A. Asynchronous full-duplex wireless. In Proceedings of the 2012 Fourth International Conference on Communication Systems and Networks (COMSNETS 2012), Bangalore, India, 3-7 January 2012; pp. 1-9.

16. Tsakalaki, E.P.; Alrabadi, O.N.; Tatomirescu, A.; De Carvalho, E.; Pedersen, G.F. Antenna cancellation for simultaneous cognitive radio communication and sensing. In Proceedings of the 2013 International Workshop on Antenna Technology (iWAT), Karlsruhe, Germany, 4-6 March 2013; pp. 215-218.

17. Choi, J.I.; Hong, S.; Jain, M.; Katti, S.; Levis, P.; Mehlman, J. Beyond full-duplex wireless. In Proceedings of the 2012 Conference Record of the Forty-Sixth Asilomar Conference on Signals, Systems, and Computers (ASILOMAR), Pacific Grove, CA, USA, 4-7 November 2012; pp. 40-44.

18. Hong, S.S.; Mehlman, J.; Katti, S. Picasso: Flexible RF and spectrum slicing. ACM SIGCOMM Comput. Commun. Rev. 2012, 42, 37-48. [CrossRef]

19. Duarte, M. Full-Duplex Wireless: Design, Implementation, and Characterization. Ph.D. Dissertation, Rice University, Houston, TX, USA, 2012.

20. He, Z.; Shao, S.; Shen, Y.; Qing, C.; Tang, Y. Performance analysis of RF self-interference cancellation in full-duplex wireless communications. IEEE Wirel. Commun. Lett. 2014, 3, 405-408. [CrossRef]

21. Duarte, M.; Dick, C.; Sabharwal, A. Experiment-driven characterization of full-duplex wireless systems. IEEE Trans. Wirel. Commun. 2012, 11, 4296-4307. [CrossRef]

22. Yang, B.; Dong, Y.; Yu, Z.; Zhou, J. An RF self-interference cancellation circuit for the full-duplex wireless communications. In Proceedings of the 2013 International Symposium on Antennas and Propagation, Nanjing, China, 23-25 October 2013; pp. 1048-1051.

23. Bharadia, D.; Katti, S. Fast-forward: Fast and constructive full-duplex relays. ACM SIGCOMM Comput. Commun. Rev. 2014, 44, 199-210. [CrossRef]

24. Riihonen, T.; Mathecken, P.; Wichman, R. Effect of oscillator phase noise and processing delay in full-duplex OFDM repeaters. In Proceedings of the 2012 Conference Record of the Forty-Sixth Asilomar Conference on Signals, Systems, and Computers (ASILOMAR), Pacific Grove, CA, USA, 4-7 November 2012; pp. 1947-1951. 
25. Dine, T.; Krishnaswamy, H. AT/R antenna pair with polarization-based reconfigurable wideband self-interference cancellation for simultaneous transmit and receive. In Proceedings of the IEEE MTT-S International Microwave Symposium, Phoenix, AZ, USA, 17-22 May 2015; pp. 1-4.

26. Debaillie, B.; van den Broek, D.J.; Lavin, C.; van Liempd, B.; Klumperink, E.A.; Palacios, C.; Craninckx, J.; Nauta, B.; Parssinen, A. Analog/RF solutions enabling compact full-duplex radios. IEEE J. Sel. Areas Commun. 2014, 32, 1662-1673. [CrossRef]

27. Khojastepour, M.A.; Sundaresan, K.; Rangarajan, S.; Zhang, X.; Barghi, S. The case for antenna cancellation for scalable full-duplex wireless communications. In Proceedings of the 10th ACM Workshop on Hot Topics in Networks, Cambridge, MA, USA, 14-15 November 2011; pp. 1-6.

28. Hu, J.; Di, B.; Liao, Y.; Bian, K.; Song, L. Hybrid MAC protocol design and optimization for full duplex Wi-Fi networks. IEEE Trans. Wirel. Commun. 2018, 17, 3615-3630. [CrossRef]

29. Alexandris, K.; Balatsoukas-Stimming, A.; Burg, A. Measurement-based characterization of residual self-interference on a full-duplex MIMO testbed. In Proceedings of the IEEE 8th Sensor Array and Multichannel Signal Processing Workshop (SAM), A Coruna, Spain, 22-25 June 2014; pp. 329-332.

30. Xu, Q.; Quan, X.; Zhang, Z.; Tang, Y.; Shen, Y. Analysis and experimental verification of digital self-interference cancelation for co-time co-frequency full-duplex LTE. Int. J. Signal Process. Image Process. Pattern Recognit. 2014, 7, 299-312. [CrossRef]

31. Razavi, B. RF Microelectronics, 2nd ed.; Prentice Hall: Hoboken, NJ, USA, 2012.

32. Bliss, D.W.; Hancock, T.; Schniter, P. Hardware phenomenological effects on cochannel full-duplex MIMO relay performance. In Proceedings of the 2012 Conference Record of the Forty-Sixth Asilomar Conference on Signals, Systems, and Computers (ASILOMAR), Pacific Grove, CA, USA, 4-7 November 2012; pp. 34-39.

33. Sahai, A.; Patel, G.; Dick, C.; Sabharwal, A. Understanding the impact of phase noise on active cancellation in wireless full-duplex. In Proceedings of the 2012 Conference Record of the Forty-Sixth Asilomar Conference on Signals, Systems, and Computers (ASILOMAR), Pacific Grove, CA, USA, 4-7 November 2012; pp. 29-33.

34. Sytjala, V.; Valkama, M.; Anttila, L.; Riihonen, T.; Korpi, D. Analysis of oscillator phase noise effects on self-interference cancellation in full-duplex OFDM radio transceivers. IEEE Trans. Wirel. Commun. 2014, 13, 2977-2990. [CrossRef]

35. Ahmed, E.; Eltawil, A.M.; Sabharwal, A. Self-interference cancellation with phase noise induced ICI suppression for full-duplex systems. In Proceedings of the 2013 IEEE Global Communications Conference (GLOBECOM), Atlanta, GA, USA, 9-13 December 2013; pp. 3384-3388.

36. Sahai, A.; Patel, G.; Dick, C.; Sabharwal, A. On the impact of phase noise on active cancelation in wireless full-duplex. IEEE Trans. Veh. Technol. 2013, 62, 4494-4510. [CrossRef]

37. Ahmed, E.; Eltawil, A.M.; Sabharwal, A. Rate gain region and design tradeoffs for full-duplex wireless communications. IEEE Trans. Wirel. Commun. 2013, 12, 3556-3565. [CrossRef]

38. Anttila, L. Digital front-end signal processing with widely linear signal models in radio devices. Ph.D. Dissertation, Tampere University of Technology, Tampere, Finland, 14 October 2011.

39. Korpi, D.; Anttila, L.; Syrjala, V.; Valkama, M. Widely linear-digital self-interference cancellation in direct-conversion full-duplex transceiver. IEEE J. Sel. Areas Commun. 2014, 32, 1674-1687. [CrossRef]

40. Li, S.; Murch, R.D. Full-duplex wireless communication using transmitter output-based echo cancellation. In Proceedings of the 2011 IEEE Global Telecommunications Conference-GLOBECOM 2011, Houston, TX, USA, 5-9 December 2011; pp. 1-5.

41. Tsai, J.H.; Chen, Y.J.; Lai, Y.F.; Shen, M.H.; Huang, P.C. A 14-bit 200MS/s current-steering DAC achieving over 82dB SFDR with digitally assisted calibration and dynamic matching techniques. In Proceedings of the Technical Program of 2012 VLSI Design, Automation and Test, Hsinchu, Taiwan, 23-25 April 2012; pp. 1-4.

42. Everett, E.; Sahai, A.; Sabharwal, A. Passive self-interference suppression for full-duplex infrastructure nodes. IEEE Trans. Wirel. Commun. 2014, 13, 680-694. [CrossRef]

43. Ahmed, E.; Eltawil, A.M.; Sabharwal, A. Self-interference cancellation with nonlinear distortion suppression for full-duplex systems. In Proceedings of the 2013 Asilomar Conference on Signals, Systems and Computers, Pacific Grove, CA, USA, 3-6 November 2013; pp. 1199-1203.

44. Bensmida, S.; Morris, K.; Lees, J.; Wright, P.; Benedikt, J.; Tasker, P.J.; Beach, M.; McGeehan, J. Power amplifier memoryless pre-distortion for 3GPP LTE application. In Proceedings of the 2009 European Microwave Conference (EuMC), Rome, Italy, 29 September-1 October 2009; pp. 1433-1436.

45. Riihonen, T.; Wichman, R. Analog and digital self-interference cai1cellation in full-duplex MIMO-OFDM transceivers with limited resolution in A/D conversion. In Proceedings of the 2012 Conference Record of the Forty-Sixth Asilomar Conference on Signals, Systems, and Computers (ASILOMAR), Pacific Grove, CA, USA, 4-7 November 2012; pp. 45-49.

46. Komatsu, K.; Miyaji, Y.; Uehara, H. Iterative Nonlinear Self-Interference Cancellation for In-Band Full-Duplex Wireless Communications Under Mixer Imbalance and Amplifier Nonlinearity. IEEE Trans. Wirel. Commun. 2020, 19, 4424-4438.

47. Evolved Universal Terrestrial Radio Access (E-UTRA). User Equipment (UE) Radio Access Capabilities 'TR 36.306'; 3rd Generation Partnership Project (3GPP): Phoenix, AZ, USA, January 2015; Available online: https://portal.3gpp.org/desktopmodules/ Specifications /SpecificationDetails.aspx?specificationId=2434 (accessed on 1 March 2021).

48. De Witt, J.J.; Van Rooyen, G.J. Novel IQ imbalance and offset compensation techniques for quadrature mixing radio transceivers. In Proceedings of the Southern African Telecommunication Networks Applications Conference (SATNAC), Stellenbosch, South Africa, 3-6 September; pp. 1-6. 
49. Panda, S.; Panigrahi, S.P.; Neema, D.D.; Mishra, R.; Sahu, M.K.; Sahu, H.S.; Panda, N.; Singh, M. A Novel Approach to Blind I/Q Mismatch and Carrier Offset Compensation. J. Signal Inf. Process. 2011, 2, 18. [CrossRef]

50. Nguyen, T.H.; Scalart, P.; Joindot, M.; Gay, M.; Bramerie, L.; Peucheret, C.; Carer, A.; Simon, J.C.; Sentieys, O. Joint simple blind IQ imbalai1ce compensation and adaptive equalization for 16-QAM optical communications. In Proceedings of the 2015 IEEE International Conference on Communications (ICC), London, UK, 8-12 June 2015; pp. 4913-4918.

51. Windisch, M.; Fettweis, G. On the performance of standard-independent I/Q imbalance compensation in OFDM direct-conversion receivers. In Proceedings of the 13th European Signal Processing Conference, Antalya, Turky, 4-8 September 2005; pp. 1-5.

52. Mailand, M.; Richter, R.; Jentschel, H.J. Blind IQ-imbalance compensation using iterative inversion for arbitrary direct conversion receivers. In Proceedings of the 14th IST Mobile and Wireless Communications Summit, Dresden, Germany, 19-23 June 2005; pp. $1-5$.

53. Özen, M.; Gustafsson, D.; Buisman, K.; Fager, C. A Doherty power amplifier design method for improved efficiency and linearity. IEEE Trans. Microw. Theory Tech. 2016, 64, 4491-4504.

54. Son, J.; Kim, I.; Moon, J.; Lee, J.; Kim, B. A highly efficient asymmetric Doherty power amplifier with a new output combining circuit. In Proceedings of the 2011 IEEE International Conference on Microwaves, Communications, Antennas and Electronic Systems (COMCAS 2011), Tel Aviv, Israel, 7-9 November 2011; pp. 1-4.

55. Moon, J.; Kim, J.; Kim, J.; Kim, I.; Kim, B. Efficiency Enhancement of Doherty Amplifier Through Mitigation of the Knee Voltage Effect. IEEE Trans. Microw. Theory Tech. 2011, 59, 143-152. [CrossRef]

56. Pasricha, R.; Sharma, S. Modeling of a Power Amplifier for Digital pre-distortion Applications using Simplified Complex Memory Polynomial. Appl. Math. Inf. Sci. 2013, 7, 1519. [CrossRef]

57. Jiang, H.; Wilford, P.A. Digital pre-distortion for power amplifiers using separable functions. IEEE Trans. Signal Process. 2010, 58, 4121-4130. [CrossRef]

58. Breed, G.; Director, E. An overview of common techniques for power amplifier linearization. IEEE Microw. Wirel. Comp. Lett. 2008, 18, 673 .

59. Tung, Y.C.; Han, S.; Chen, D.; Shin, K.G. Vulnerability and protection of channel state information in multiuser MIMO networks. In Proceedings of the 2014 ACM SIGSAC Conference on Computer and Communications Security, Scottsdale, AZ, USA, 3-7 November 2014; pp. 775-786.

60. Sharma, M. Effective Channel State Information (CSI) Feedback for MIMO Systems in Wireless Broadband Communications. Ph.D. Thesis, Queensland University of Technology, Queensland, Australia, 2014.

61. Cho, Y.S.; Kim, J.; Yang, W.Y.; Kang, C.G. MIMO-OFDM Wireless Communications with MATLAB; John Wiley and Sons: Hoboken, NJ, USA, 2010.

62. Campi, M.; Leonardi, R.; Rossi, L.A. Generalized super-exponential method for blind equalization using Kautz filters. In Proceedings of the IEEE Signal Processing Workshop on Higher-Order Statistics (SPW-HOS '99), Caesarea, Israel, 16 June 1999; pp. 107-111.

63. e Silva, T.O. On the adaptation of the pole of Laguerre-lattice filters. In Proceedings of the 8th European Signal Processing Conference (EUSIPCO 1996), Trieste, Italy, 10-13 September 1996; pp. 1-4.

64. Kim, S.W.; Park, Y.C.; Youn, D.H. A variable step-size filtered-x gradient adaptive lattice algorithm for active noise control. In Proceedings of the 2012 IEEE International Conference on Acoustics, Speech and Signal Processing (ICASSP), Kyoto, Japan, 25-30 March 2012; pp. 189-192.

65. Dam, H.; Cantoni, A.; Nordholm, S.; Teo, K.L. Digital Laguerre filter design with maximum passband-to-stopband energy ratio subject to peak and group delay constraints. IEEE Trans. Circuits Syst. I Regul. Pap. 2006, 53, 1108-1118. [CrossRef]

66. Paatero, T.; Karjalainen, M.; Harma, A. Modeling, and equalization of audio systems using Kautz filters. In Proceedings of the 2001 IEEE International Conference on Acoustics, Speech, and Signal Processing, Proceedings (Cat. No. 01CH37221), Salt Lake City, UT, USA, 7-11 May 2001; pp. 3313-3316.

67. Kurt, T.; Lerbour, R.; Le Helloco, Y.; Breton, B. Adaptive Kalman filtering for local mean power estimation in mobile communications. In Proceedings of the IEEE Vehicular Technology Conference (VTC Fall), Montreal, QC, Canada, 25-28 September 2006; pp. 1-4.

68. Lekshmi, B.; Susan, S.; Apren, D.T. Channel Estimation with Extended Kalman Filter for Fading Charmels. Int. J. Electron. Commun. Comput. Technol. 2013, 3, 9-13.

69. Ayesha, A.; Chaudhry, S.M. Self-Interference Cancellation for Full-Duplex Radio Transceivers Using Extended Kalman Filter. Natl. Acad. Sci. Lett. 2020, 7, 631-634. [CrossRef]

70. Mohinder, S.; Angus, P. Kalman Filtering: Theory and Practice Using Matlab; John Wiley and Sons: Hoboken, NJ, USA, 2001.

71. Anderson, B.D.; Moore, J.B. Optimal Filtering; Dover Publication, Inc.: Mineola, NY, USA, 2012.

72. Chen, L.; Mercorelli, P.; Liu, S. A Kalman estimator for detecting repetitive disturbances. In Proceedings of the 2005, American Control Conference, Portland, OR, USA, 8-10 June 2005; pp. 1631-1636.

73. Faragher, R. Understanding the basis of the Kalman filter via a simple and intuitive derivation [lecture notes]. IEEE Signal Process. Mag. 2012, 29, 128-132. [CrossRef]

74. Duarte, M.; Sabharwal, A.; Aggarwal, V.; Jana, R.; Ramakrishnan, K.K.; Rice, C.W.; Shankaranarayanan, N.K. Design and characterization of a full-duplex multiantenna system for WiFi networks. IEEE Trans. Veh. Technol. 2014, 3, 1160-1177. [CrossRef] 
75. Heino, M.; Korpi, D.; Huusari, T.; Rodriguez, E.A.; Venkatasubramanian, S.; Riihonen, T.; Anttila, L.; Icheln, C.; Haneda, K.; Wichman, R. Recent advances in antenna design and interference cancellation algorithms for in-band full duplex relays. IEEE Commun. Mag. 2015, 5, 91-101. [CrossRef]

76. Huusari, T.; Choi, Y.S.; Liikkanen, P.; Korpi, D.; Talwar, S.; Valkama, M. Wideband self-adaptive RF cancellation circuit for full-duplex radio: Operating principle and measurements. In Proceedings of the 81st Vehicular Technology Conference (VTC Spring), Glasgow, UK, 11-14 May 2015; pp. 1-7.

77. Tamminen, J.; Turunen, M.; Korpi, D.; Huusari, T.; Choi, Y.-S.; Talwar, S.; Valkama, M. Digitally-controlled RF self-interference canceller for full-duplex radios. In Proceedings of the 24th European Signal Processing Conference (EUSIPCO), Budapest, Hungary, 29 August-2 September 2016; pp. 783-787.

78. Khaledian, S.; Farzami, F.; Smida, B.; Erricolo, D. Inherent self-interference cancellation at $900 \mathrm{MHz}$ for in-band full-duplex applications. In Proceedings of the IEEE 19th Wireless and Microwave Technology Conference (WAMICON), Sand Key, FL, USA, 9-10 April 2018; pp. 1-4.

79. Kiayani, A.; Waheed, M.Z.; Anttila, L.; Abdelaziz, M.; Korpi, D.; Syrjala, V.; Kosunen, M.; Stadius, K.; Ryynänen, J.; Valkama, M. Adaptive nonlinear RF cancellation for improved isolation in simultaneous transmit-receive systems. IEEE Trans. Microw. Theory Tech. 2018, 5, 2299-2312. [CrossRef]

80. Islam, M.A.; Smida, B. A comprehensive self-interference model for single-antenna full-duplex communication systems. In Proceedings of the IEEE International Conference on Communications (ICC 2019), Shanghai, China, 20-24 May 2019; pp. 1-7.

81. Martínez, M.F.; Alonso, F.J.M.; Valcarce, R.L. Solving Self-Interference Issues in a Full-Duplex Radio Transceiver. Multidiscip. Digit. Publ. Inst. Proc. 2019, 1, 35.

82. Singh, V.; Mondal, S.; Gadre, A.; Srivastava, M.; Paramesh, J.; Kumar, S. Millimeter-wave full duplex radios. In Proceedings of the 26th Annual International Conference on Mobile Computing and Networking (MobiCom '20), London, UK, 21-25 September 2020; pp. 1-14. 